\title{
Trait Anxiety in Individuals on the Autism Spectrum: a Systematic Review
}

\author{
Rebecca Jolliffe ${ }^{1,2} \cdot$ Dawn Adams $^{1,2} \cdot$ Kate Simpson $^{1,2}$
}

Received: 26 October 2021 / Accepted: 5 February 2022

(c) The Author(s) 2022

\begin{abstract}
Although anxiety is commonly researched in autism, the focus has predominantly been on anxiety disorders and not upon general levels of anxiousness or trait anxiety. This review summarises research investigating trait anxiety in autism. Systematic searches yielded 1099 records, with 23 studies meeting inclusion criteria. Study participants were mainly males from Western countries, with no representation of older adults or individuals with intellectual disability. All articles used self-report questionnaire trait anxiety measures. Anxiety measure psychometric details were absent in most studies, with 21 using subjective measures that are not validated for use in autism. Results showed higher trait anxiety scores in autism versus control groups, and correlations between trait anxiety scores and other study outcomes.
\end{abstract}

Keywords Anxious $\cdot$ State-trait $\cdot$ Autistic $\cdot$ Adult $\cdot$ Child

\section{Introduction}

Autism spectrum disorder (ASD; hereafter autism) is a neurodevelopmental condition characterised by differences in two domains: social communication and interaction, encompassing social reciprocity, non-verbal communication, and relationships, and restricted and repetitive behaviours, comprising stereotyped or repetitive behaviours, insistence on sameness, circumscribed interests, and sensory responsivity (American Psychiatric Association 2013). Co-occurring mental health disorders are frequently reported by individuals on the autism spectrum, with clinically elevated anxiety having a lifetime prevalence of $42 \%$ (Hollocks et al., 2019).

\section{Trait Anxiety in Autism}

Anxiety is the feeling of distress or apprehension that is experienced in response to an unspecified or uncertain threat. These anxious feelings occur on a continuum across

Dawn Adams

Dawn.Adams@Griffith.edu.au

1 Autism Centre of Excellence, School of Education and Professional Studies, Griffith University, Messines Ridge Road, Mount Gravatt, Brisbane, QLD 4122, Australia

2 Griffith Institute of Educational Research, Griffith University, Brisbane, Australia normal and abnormal psychology, where anxiety disorders may be diagnosed when anxious feelings are atypical in their activation or duration (American Psychiatric Association, 2013). However, anxiety often occurs outside of the presence of an anxiety disorder. The state-trait anxiety model describes the difference between the transient anxiety felt in response to an immediate stressor (state anxiety) and a person's general personality trait of anxiety proneness (trait anxiety; Spielberger, 1966). The distinction between state and trait anxiety has been extensively supported in studies involving neurotypical participants who show differences in genetic and environmental influences (Lau et al., 2006), different neurological structural and functional patterns (Saviola et al., 2020), and disparate physiological responses to stress (Jonsson, 2007). Spielberger (1966) proposed that trait and state anxiety can be related, in that a person high in trait anxiety is more likely to perceive a situation as threatening, and therefore more likely to experience high state anxiety. This is supported in studies that showed neurotypical individuals with high trait anxiety responding with higher levels of state anxiety in standardised stress tasks (Houston et al., 1984; Villada et al., 2016). High trait anxiety can also induce an experience of chronically high state anxiety, thus causing a prolonged stress response that has been associated with the development of mood and anxiety disorders (McEwen \& Gianaros, 2011). 
Although the state-trait model of anxiety has been extensively investigated in neurotypical research, its exploration in individuals on the autism spectrum is relatively limited. One study which investigated state and trait anxiety did so with a sample of 22 adolescents on the autism spectrum compared with 32 neurotypical peers (Mertens et al., 2017). Trait anxiety was assessed via an array of self- and parentreported standardised questionnaires, while several physiological arousal markers were measured to assess state anxiety during stress tasks. Parent- and self-reported trait anxiety levels were higher for the autism sample for some, but not all, of the standardised questionnaires, indicating the importance of testing instrument validity in this population. These higher levels of trait anxiety in adolescents on the autism spectrum reflect similar findings in child and adult studies (Corbett et al., 2019; Zukerman et al., 2019b). Mertens et al. (2017) also identified good agreement between parent- and self-reported trait anxiety levels in participants on the autism spectrum, but, due to lower participant numbers during physiological testings, were unable to conduct correlational analysis between trait anxiety results and physiological measures.

\section{Measurement of Anxiety in Autism}

In the research of anxiety in autism, it is important to consider some of the challenges in measurement and diagnosis. Although studies of anxiety in children and adolescents on the spectrum preferentially include parentreport over self-report methods (van Steensel \& Heeman, 2017), there are limitations to informant-report methods. Anxiety is an internal experience; however, parents and other informants are only able to provide data on externally observable aspects of anxiety (Mazzone et al., 2012) or internal experiences that the person has informed them of. Research investigating anxiety measurement in individuals on the autism spectrum has shown that severity ratings can differ between respondent and across settings (Adams, Clark, et al., 2019; Adams, Young, et al., 2019; Kanne et al., 2009; Stratis \& Lecavalier, 2015), reinforcing the importance of study designs that include reports from multiple informants. This may be particularly important for trait anxiety, given that it is reflective of a general personality trait rather than specific moments or experiences of anxiety.

\section{This study}

The heterogeneity of anxiety presentation and high prevalence of anxiety disorders across the lifespan of individuals on the autism spectrum indicate the need for examination of trait anxiety in this population. To better understand the current knowledge of trait anxiety in individuals on the autism spectrum, the aim of this review was to gather the research completed to date, outline trends in the research, and identify areas for future study. The following research questions were posed:

1) What are the characteristics and outcomes of research that has explored trait anxiety in individuals on the autism spectrum?

2) What is the quality of the existing research on trait anxiety in individuals on the autism spectrum?

3) Which methods have been used to measure trait anxiety in individuals on the autism spectrum?

4) What are the psychometric properties of the measures used and to what extent are they reliable and valid for use for those with a diagnosis of autism?

\section{Method}

\section{Protocol and Registration}

The method of analysis and the inclusion criteria for this systematic review were specified in advance in a protocol that was registered online with the international prospective register of systematic reviews (PROSPERO; registration number: CRD42020207483). Preferred Reporting Items for Systematic Reviews and Meta-Analyses (PRISMA; Page et al., 2021) standards were followed in all stages of this systematic review.

\section{Eligibility Criteria}

PRISMA guidelines (Page et al., 2021) outline the importance of inclusion criteria that identify well-defined groups and outcomes. The inclusion criteria for this review were as follows: (1) the article reports an original study published in English in a peer-reviewed journal between 1994 and 2020 reporting on human participants, (2) the study reports that a reliable diagnostic or screening measure of autism was utilised to confirm autism diagnosis, (3) the study reports on a measure that the authors of the study specify to have been used to represent trait anxiety, and (4) data from the measure of trait anxiety for those on the autism spectrum are presented separately from any other group included in the study.

Studies were excluded from the review based on one or more of the following criteria: (1) no reliable diagnostic or screening measure was performed during the study or recruitment process to confirm autism diagnosis, (2) the author of the article did not identify that the type of anxiety being measured was trait anxiety, (3) trait anxiety was not measured in the individual with the autism diagnosis, and (4) trait anxiety results of the autism group were not presented separately. 
One objective of this review was to identify and characterise the original research that has studied trait anxiety in individuals on the autism spectrum, so reviews, meta-analyses, grey literature, opinion pieces, dissertations, editorials, book chapters, and conference proceedings were excluded. Case studies, single case designs, and descriptive pieces were also excluded from this review due to the increased risk of bias and decreased generalisability associated with studies of a very small sample size. The search was limited to those articles published after 1994, the year of publication of the Diagnostic and Statistical Manual of Mental Disorders, 4th ed. (American Psychiatric Association, 1994). This allowed for consistent interpretation of the language used for diagnoses of the participants sampled. To align with the encompassing nature of this review to characterise the research available, no restrictions were made regarding age, gender, ethnicity, co-morbidities, treatment, or medication status of participants. Nor were any restrictions placed on the other outcomes investigated by the studies, their geographical location, or their study design.

\section{Search Strategy}

In September 2020, eight databases (CINAHL, Embase, ERIC, MEDLINE, ProQuest Central, PsycINFO, Scopus, and Web of Science) were searched for studies measuring trait anxiety in participants on the autism spectrum. A second search was completed in March 2021 to update results of the initial search. Filters were applied to incorporate only peer-reviewed journal articles, written in English, that were published from 1994 to 2020 inclusive. Databases were searched using the search string (autis* OR asd OR asc OR asperger* OR "pervasive development* disorder*" OR pdd OR hfa) AND (trait NEAR/10 anxi*). Keyword structure and proximity operators were adjusted according to the specifications of each database, and, when available, expanders mapping to preferred or equivalent terms were added. To most closely align the search locations of the databases used, the search of Scopus through Elsevier applied the TITLEABS-KEY field tag, the search of Web of Science through Clarivate Analytics applied the TS field tag, and the search of ProQuest Central and ERIC through ProQuest used the NOFT field tag. All other databases employed the default search locations.

\section{Study Selection Process}

Electronic searches yielded 1099 records (see Fig. 1). Following the removal of duplicates, the primary rater screened the titles and abstracts of 451 articles for inclusion in fulltext screening. A second rater, blinded to the ratings of the primary rater, assessed a random sample of $20 \%$ of the reports. Cohen's Kappa showed strong inter-rater reliability
( $\kappa=0.82$; McHugh, 2012). Any report that was considered to meet inclusion criteria by either rater was then included in full-text screening. All 296 articles identified for full-text review were assessed independently by both raters. Interrater reliability was calculated using Cohen's Kappa and again showed a strong $(\kappa=0.84)$ agreement. Discussion of the eight articles where ratings disagreed resulted in consensus agreement on six articles. A third rater was consulted for the remaining two studies. A total of 23 articles met the criteria for inclusion in this review. There were four studies included in this review that met inclusion criteria based on additional information, regarding diagnostic procedures or the anxiety measure used, requested directly from the corresponding authors and supplied via email.

\section{Data Extraction}

Extracted data included the details of the research team (including geographical location of the study), study details (study design type and any physiological measure, test, or intervention completed by study groups), study group participant characteristics (sample number, age, gender, IQ, medication use, and race/ethnicity of both the autism sample and any control or comparison groups, as well as the autism subtype and diagnostic/screening measure used for the autism group), details of the trait anxiety measure (scale/ subscale, informant, and psychometric properties reported), and the main trait anxiety-related outcomes reported. The results of primary interest were scores on measures of trait anxiety, between- or within-group analysis of trait anxiety outcomes, and statistical analyses with trait anxiety data. Data were independently extracted to an Excel spreadsheet by two researchers, and percentage agreement calculation across all included studies showed strong (88.96\%) agreement. Disagreements in extracted data were discussed and consensus was reached in all cases.

\section{Risk of Bias in Individual Studies}

The quality of included articles was assessed by the primary researcher. The Strengthening the Reporting of Observational Studies in Epidemiology (STROBE; von Elm et al., 2007) checklist was used to evaluate observational studies, and the Consolidated Standards of Reporting Trials (CONSORT; Schulz et al., 2010) checklist was used to evaluate interventional studies. Each article was assessed for which checklist items were adequately reported (as characterised in the explanation and elaboration publications for each checklist; Moher et al., 2010; Vandenbroucke et al., 2007). With subitems included, the total number of items on the STROBE checklist was 34 , and on the CONSORT checklist was 37 . Some checklist items were specific for only one study design type (e.g. case-control or randomised trial), 
Fig. 1 PRISMA flow diagram for review process. From: Page et al. (2021). The PRISMA 2020 statement: An updated guideline for reporting systematic reviews. BMJ, 372, n71. 10.1136/bmj.n71. For more information, visit: http://www. prisma-statement.org/

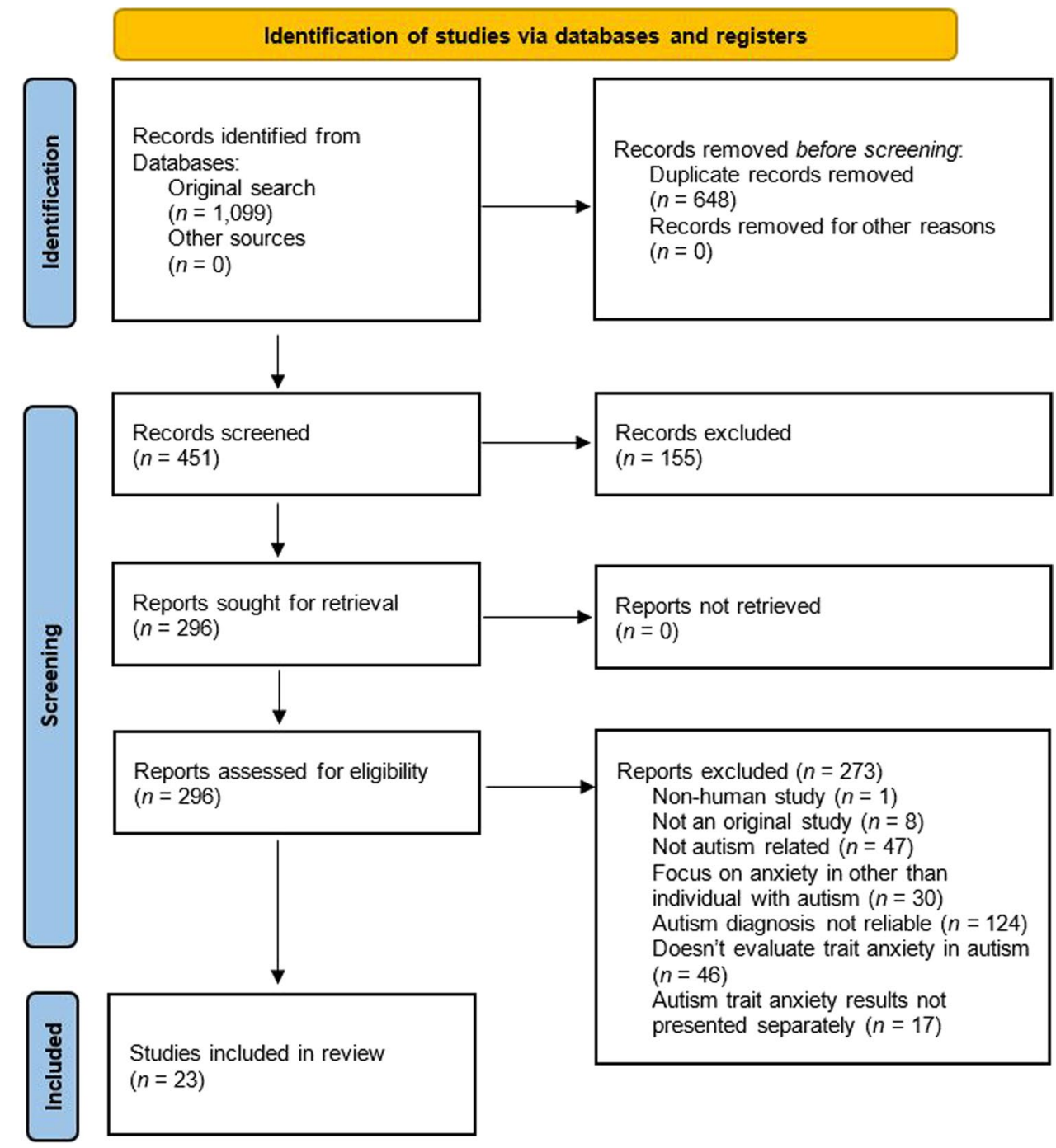

and therefore, a rating of "not applicable" was noted if the item did not align with the study design of the article being assessed. Where the primary researcher was undecided on the rating of any item, the item was discussed with a second rater and a consensus was reached in all cases. A global quality score was determined for each article (see Table 1) to indicate the number of adequately reported items compared with the total items applicable to the study, with a higher score representing stronger reporting quality.

\section{Results}

\section{Study Characteristics}

A total of 23 articles met selection criteria for inclusion; details of each study are presented in Table 1. The articles were published by 14 research groups, with most studies conducted in the USA (47.8\%) and the UK $(21.7 \%)$. The remaining studies were from Israel $(8.7 \%)$, two studies that recruited participants across multiple European countries (8.7\%), Canada (4.3\%), the Netherlands (4.3\%), and South Korea (4.3\%). Most studies (82.6\%) were observational in nature and involved a cross-sectional study design. The remaining four studies $(17.4 \%)$ implemented a randomised control trial (RCT) design. Of these, two used a theatre-based treatment (Corbett et al., 2017; Ioannou et al., 2020) and one employed a social skills training program (Yoo et al., 2014), evaluating the effect of the intervention on trait anxiety scores. In the fourth RCT study, Althaus et al. (2015) measured trait anxiety scores only at baseline and used this data in subsequent analyses; therefore, the data reported that are relevant to the current review were cross-sectional in nature. As such, this focus on pre-interventional data means that the study will be discussed alongside the observational studies for the remainder of the review. Therefore, this review will discuss 20 observational and three interventional studies. 
Table 1 Study characteristics of included articles

\begin{tabular}{|c|c|c|c|c|c|c|c|c|}
\hline \multirow{2}{*}{$\begin{array}{l}\text { Study refer- } \\
\text { ence }\end{array}$} & \multicolumn{5}{|c|}{ Participant characteristics } & \multirow{2}{*}{$\begin{array}{l}\text { Autism sub- } \\
\text { type (in-study } \\
\text { measures) }\end{array}$} & \multirow[t]{2}{*}{ Study design } & \multirow{2}{*}{$\begin{array}{l}\text { Reporting } \\
\text { score }\end{array}$} \\
\hline & Study groups & $\begin{array}{l}\text { Age in years } \\
\text { Mean }(S D) \\
\text { range }\end{array}$ & Male (\%) & $\begin{array}{l}\text { FSIQ } \\
\text { Mean }(S D)\end{array}$ & $\begin{array}{l}\text { Medication } \\
\text { status }\end{array}$ & & & \\
\hline \multirow[t]{2}{*}{$\begin{array}{l}\text { Althaus et al. } \\
\text { (2015) }\end{array}$} & \multirow{2}{*}{$\begin{aligned} & \text { Autism }(N=3 \\
& \text { NT }(N=3\end{aligned}$} & $\begin{array}{l}22.69(4.83) \\
18-34\end{array}$ & 100 & $\begin{array}{r}104.97 \\
(17.49)\end{array}$ & Unmedicated & \multirow{2}{*}{$\begin{array}{l}\text { Autistic } \\
\text { disorder, } \\
\text { Asperger's } \\
\text { disorder, } \\
\text { PDD-NOS } \\
\text { (ADOS, AQ) }\end{array}$} & \multirow[t]{2}{*}{$\mathrm{O}$} & \multirow[t]{2}{*}{$\begin{array}{l}\text { 22/32 (69\%) } \\
\text { STROBE }\end{array}$} \\
\hline & & $\begin{array}{l}22.60(3.21) \\
18-31\end{array}$ & 100 & $\begin{array}{l}104.38 \\
(10.25)\end{array}$ & Unmedicated & & & \\
\hline \multirow[t]{2}{*}{$\begin{array}{l}\text { Bernier et al. } \\
(2005)\end{array}$} & \multicolumn{2}{|c|}{$\begin{array}{c}\text { Autism }(N=14) 18.40(5.50) \\
12-45\end{array}$} & 85.7 & $106.1(13.0)$ & NR & \multirow{2}{*}{$\begin{array}{l}\text { ASD } \\
\text { (ADOS, ADI- } \\
\text { R) }\end{array}$} & \multirow[t]{2}{*}{$\mathrm{O}$} & \multirow[t]{2}{*}{$\begin{array}{l}10 / 28(36 \%) \\
\text { STROBE }\end{array}$} \\
\hline & $\mathrm{NT}(N=1$ & $\begin{array}{l}19.70(8.20) \\
12-45\end{array}$ & 85.7 & $114.9(12.5)$ & NR & & & \\
\hline \multirow[t]{2}{*}{$\begin{array}{l}\text { Corbett et al. } \\
(2017)\end{array}$} & $\begin{array}{l}\text { Autism IN } \\
(n=17)\end{array}$ & $\begin{array}{l}\text { T } 11.27(2.51) \\
8-14\end{array}$ & 76.5 & $\begin{array}{l}106.06 \\
(16.83)\end{array}$ & $\begin{array}{l}58.8 \% \text { medi- } \\
\text { cated }\end{array}$ & \multirow[t]{2}{*}{$\begin{array}{l}\text { ASD } \\
\text { (ADOS) }\end{array}$} & \multirow[t]{2}{*}{$\mathrm{I}$} & \multirow[t]{2}{*}{$\begin{array}{l}16 / 32(50 \%) \\
\text { CONSORT }\end{array}$} \\
\hline & $\begin{array}{l}\text { Autism WL } \\
(n=13)\end{array}$ & $\begin{array}{l}\text { C } 10.74(1.89) \\
8-14\end{array}$ & 84.6 & 95.85 (21.19) & $\begin{array}{l}69.2 \% \text { medi- } \\
\text { cated }\end{array}$ & & & \\
\hline \multirow[t]{2}{*}{$\begin{array}{l}\text { Corbett et al. } \\
\text { (2019) }\end{array}$} & Autism $(N=3$ & $\begin{array}{l}11.17(1.08) \\
10-12\end{array}$ & 64.5 & $98.29(20.27)$ & NR & \multirow[t]{2}{*}{$\begin{array}{l}\text { ASD } \\
(\text { ADOS-2) }\end{array}$} & \multirow[t]{2}{*}{$\mathrm{O}$} & \multirow[t]{2}{*}{$\begin{array}{l}\text { 17/29 (59\%) } \\
\text { STROBE }\end{array}$} \\
\hline & NT $(N=2$ & $\begin{array}{l}11.09(0.91) \\
10-12\end{array}$ & 72 & $\begin{array}{l}113.76 \\
(16.57)\end{array}$ & NR & & & \\
\hline \multirow[t]{2}{*}{$\begin{array}{l}\text { Edmiston } \\
\text { et al. (2017) }\end{array}$} & Autism $(N=2$ & ) 14.8 (1.36) & 100 & $\begin{array}{l}107.71 \\
(22.03)\end{array}$ & NR & \multirow{2}{*}{$\begin{array}{l}\text { ASD } \\
\text { (ADOS-2, } \\
\text { ADI-R, } \\
\text { SCQ, SRS- } \\
\text { 2) }\end{array}$} & \multirow[t]{2}{*}{$\mathrm{O}$} & \multirow[t]{2}{*}{$\begin{array}{l}\text { 13/48 (46\%) } \\
\text { STROBE }\end{array}$} \\
\hline & $\mathrm{NT}(N=1$ & ) $14.99(1.52)$ & 100 & $\begin{array}{c}113.86 \\
(13.44)\end{array}$ & NR & & & \\
\hline \multirow[t]{2}{*}{$\begin{array}{l}\text { Ioannou et al. } \\
(2020)\end{array}$} & $\begin{array}{l}\text { Autism IN } \\
(n=44)\end{array}$ & $\begin{array}{l}\text { T Whole sample } \\
8-16\end{array}$ & $\begin{array}{l}\text { Whole sample } \\
76.6\end{array}$ & $\begin{array}{l}\text { Whole sample } \\
\mathrm{IQ} \geq 70\end{array}$ & NR & \multirow[t]{2}{*}{$\begin{array}{l}\text { ASD } \\
(\text { ADOS-2) }\end{array}$} & \multirow[t]{2}{*}{ I } & $\begin{array}{l}16 / 31(52 \%) \\
\text { CONSORT }\end{array}$ \\
\hline & $\begin{array}{l}\text { Autism WL } \\
(n=33)\end{array}$ & & & & NR & & & \\
\hline \multirow[t]{2}{*}{$\begin{array}{l}\text { Lanni et al. } \\
\text { (2012) }\end{array}$} & Autism $(N=1$ & $\begin{array}{l}9.77(1.26) \\
8-12\end{array}$ & 100 & 92.77 (12.29) & Unmedicated & \multirow[t]{2}{*}{$\begin{array}{l}\text { Autism } \\
\text { (ADOS) }\end{array}$} & \multirow[t]{2}{*}{$\mathrm{O}$} & $\begin{array}{l}11 / 29(38 \%) \\
\text { STROBE }\end{array}$ \\
\hline & $\mathrm{NT}(N=1$ & $\begin{array}{l}9.55(1.65) \\
8-12\end{array}$ & 100 & $124.87(9.17)$ & Unmedicated & & & \\
\hline $\begin{array}{l}\text { Lassalle et al. } \\
\text { (2017) }\end{array}$ & Autism $(N=2$ & $\begin{array}{l}23.63(9.86) \\
9-43\end{array}$ & 100 & $\begin{array}{l}113.15 \\
(12.36)\end{array}$ & NR & $\begin{array}{l}\text { ASD } \\
\text { (ADOS, ADI- }\end{array}$ & $\mathrm{O}$ & $\begin{array}{l}15 / 29(52 \%) \\
\text { STROBE }\end{array}$ \\
\hline & NT $(N=2$ & $\begin{array}{l}19.7(7.74) \\
12-43\end{array}$ & 100 & $112.0(13.73)$ & NR & R, DISCO) & & \\
\hline $\begin{array}{l}\text { Lassalle et al. } \\
\text { (2019) }\end{array}$ & Autism $(N=1$ & $\begin{array}{l}25.27(8.83) \\
15-43\end{array}$ & 89.5 & $111.21(8.83)$ & NR & $\begin{array}{l}\text { ASD, } \\
\text { Asperger's }\end{array}$ & $\mathrm{O}$ & $\begin{array}{l}16 / 28(57 \%) \\
\text { STROBE }\end{array}$ \\
\hline & $\mathrm{NT}(N=2$ & $\begin{array}{l}24.15(7.57) \\
15-43\end{array}$ & 85 & $111.85(9.15)$ & NR & $\begin{array}{l}\text { syndrome, } \\
\text { PDD-NOS } \\
\text { (ADOS, ADI- } \\
\text { R, AQ) }\end{array}$ & & \\
\hline $\begin{array}{l}\text { Limoges et al. } \\
\text { (2005) }\end{array}$ & Autism $(N=1$ & $\begin{array}{l}22.1(3.6) \\
16-27\end{array}$ & 93.7 & $102.1(10.3)$ & Unmedicated & $\begin{array}{l}\text { High- } \\
\text { functioning }\end{array}$ & $\mathrm{O}$ & $\begin{array}{l}13 / 30(43 \%) \\
\text { STROBE }\end{array}$ \\
\hline & $\mathrm{NT}(N=1$ & $\begin{array}{l}20.6(3.9) \\
16-26\end{array}$ & 93.7 & $114.4(14.2)$ & Unmedicated & $\begin{array}{l}\text { autism, } \\
\text { Asperger's } \\
\text { syndrome } \\
\text { (ADI-R) }\end{array}$ & & \\
\hline Maras and & Autism $(N=3$ & $39.44(12.35)$ & 75 & $113(15.86)$ & NR & ASD & $\mathrm{O}$ & $14 / 28(50 \%)$ \\
\hline $\begin{array}{l}\text { Bowler } \\
(2012)\end{array}$ & $\mathrm{NT}(N=3$ & $42.03(12.45)$ & 73.3 & $\begin{array}{l}107.93 \\
(15.13)\end{array}$ & NR & (ADOS, AQ) & & STROBE \\
\hline
\end{tabular}


Table 1 (continued)

\begin{tabular}{|c|c|c|c|c|c|c|c|c|}
\hline \multirow{2}{*}{$\begin{array}{l}\text { Study refer- } \\
\text { ence }\end{array}$} & \multicolumn{5}{|c|}{ Participant characteristics } & \multirow{2}{*}{$\begin{array}{l}\text { Autism sub- } \\
\text { type (in-study } \\
\text { measures) }\end{array}$} & \multirow[t]{2}{*}{ Study design } & \multirow{2}{*}{$\begin{array}{l}\text { Reporting } \\
\text { score }\end{array}$} \\
\hline & Study groups & $\begin{array}{l}\text { Age in years } \\
\text { Mean }(S D) \\
\text { range }\end{array}$ & Male (\%) & $\begin{array}{l}\text { FSIQ } \\
\text { Mean }(S D)\end{array}$ & $\begin{array}{l}\text { Medication } \\
\text { status }\end{array}$ & & & \\
\hline \multirow[t]{2}{*}{$\begin{array}{l}\text { Mertens et al. } \\
\text { (2017) }\end{array}$} & Autism $(N=22$ & $13.8(2.2)$ & 81.8 & $111.8(20.7)$ & $\begin{array}{l}22.7 \% \text { medi- } \\
\text { cated }\end{array}$ & \multirow[t]{2}{*}{$\begin{array}{l}\text { ASD } \\
\text { (ADOS-2) }\end{array}$} & \multirow[t]{2}{*}{$\mathrm{O}$} & $\begin{array}{l}15 / 29(52 \%) \\
\text { STROBE }\end{array}$ \\
\hline & $\mathrm{NT}(N=32$ & $13.2(2.0)$ & 68.7 & $106.4(13.5)$ & $\begin{array}{l}3.1 \% \text { medi- } \\
\text { cated }\end{array}$ & & & \\
\hline \multirow[t]{2}{*}{$\begin{array}{l}\text { Mikita et al. } \\
\text { (2015) }\end{array}$} & Autism $(N=52$ & $\begin{array}{l}12.8(2.0) \\
10-16\end{array}$ & 100 & $101.2(13.5)$ & Unmedicated & \multirow{2}{*}{$\begin{array}{l}\text { High-func- } \\
\text { tioning ASD } \\
\text { (ADOS, ADI- } \\
\text { R, SCQ) }\end{array}$} & \multirow[t]{2}{*}{$\mathrm{O}$} & $\begin{array}{l}\text { 16/30 (53\%) } \\
\text { STROBE }\end{array}$ \\
\hline & $\mathrm{NT}(N=23$ & $\begin{array}{l}13.9(1.9) \\
10-16\end{array}$ & 100 & $117.7(9.1)$ & Unmedicated & & & \\
\hline \multirow[t]{3}{*}{$\begin{array}{l}\text { Milne et al. } \\
\text { (2019) }\end{array}$} & $\begin{array}{l}\text { Whole sample } \\
(N=99)\end{array}$ & e 37.5 (13.5) & 48.5 & NR & $\begin{array}{l}19.2 \% \text { medi- } \\
\text { cated }\end{array}$ & \multirow[t]{3}{*}{$\begin{array}{l}\text { ASD } \\
\text { (RAADS-R) }\end{array}$} & \multirow[t]{3}{*}{$\mathrm{O}$} & $\begin{array}{l}24 / 31(77 \%) \\
\text { STROBE }\end{array}$ \\
\hline & $\begin{array}{l}\text { Unmedicatec } \\
\text { autism }(n=22)\end{array}$ & d 42.1 (14.0) & 59.1 & NR & Unmedicated & & & \\
\hline & $\begin{array}{l}\text { Unmedicated NT } \\
(n=22)\end{array}$ & Г 37.2 (10.7) & 45.5 & NR & Unmedicated & & & \\
\hline \multirow[t]{2}{*}{$\begin{array}{l}\text { Milosavljevic } \\
\text { et al. (2016) }\end{array}$} & $\begin{array}{l}\text { Autism - ALX } \\
(n=25)\end{array}$ & $\begin{array}{r}15.41(0.45) \\
14.75-16.75\end{array}$ & 96 & $95.52(10.60)$ & NR & \multirow{2}{*}{$\begin{array}{l}\text { ASD } \\
\text { (ADOS, ADI- } \\
\text { R) }\end{array}$} & \multirow[t]{2}{*}{$\mathrm{O}$} & \multirow[t]{2}{*}{$\begin{array}{l}\text { 18/30 (60\%) } \\
\text { STROBE }\end{array}$} \\
\hline & $\underset{(n=31)}{\text { Autism }+ \text { ALX }}$ & $\begin{array}{l}\mathrm{X} 15.49(0.5) \\
14.67-16.75\end{array}$ & 96.8 & $92.77(12.45)$ & NR & & & \\
\hline \multirow[t]{2}{*}{$\begin{array}{l}\text { Paul et al. } \\
\text { (2014) }\end{array}$} & Autism $(N=28$ & $\begin{array}{l}29.61(11.11) \\
19-56\end{array}$ & 82.1 & $\begin{array}{c}109.54 \\
(11.86)\end{array}$ & NR & \multirow{2}{*}{$\begin{array}{l}\text { Autism, } \\
\text { Asperger's } \\
\text { syndrome } \\
\text { (ADOS, ADI- } \\
\text { R, SCQ) }\end{array}$} & \multirow[t]{2}{*}{$\mathrm{O}$} & \multirow[t]{2}{*}{$\begin{array}{l}16 / 30(53 \%) \\
\text { STROBE }\end{array}$} \\
\hline & $\operatorname{AgCC}(N=26$ & $\begin{array}{l}28.77(11.25) \\
16-54\end{array}$ & 61.5 & $95.46(14.15)$ & NR & & & \\
\hline \multirow{2}{*}{$\begin{array}{l}\text { Sebastian } \\
\text { et al. (2009) }\end{array}$} & Autism $(n=13$ & ) $16.9(0.3)$ & 100 & $100.46(7.49)$ & NR & \multirow{2}{*}{$\begin{array}{l}\text { Childhood } \\
\text { autism, PDD } \\
\text { other, PDD } \\
\text { unspecified } \\
\text { (ADOS, ADI- } \\
\text { R) }\end{array}$} & \multirow[t]{2}{*}{$\mathrm{O}$} & $16 / 29(55 \%)$ \\
\hline & $\mathrm{NT}(N=13$ & ) $16.9(0.7)$ & 100 & $102.46(8.62)$ & NR & & & STROBE \\
\hline \multirow{2}{*}{$\begin{array}{l}\text { Simon and } \\
\text { Corbett } \\
(2013)\end{array}$} & Autism $(N=19$ & $\begin{array}{l}9.99(1.29) \\
8-12\end{array}$ & 100 & $98.56(17.95)$ & Unmedicated & \multirow{2}{*}{$\begin{array}{l}\text { Autistic dis- } \\
\text { order } \\
\text { (ADOS) }\end{array}$} & \multirow[t]{2}{*}{$\mathrm{O}$} & $\begin{array}{l}14 / 28(50 \%) \\
\text { STROBE }\end{array}$ \\
\hline & $\mathrm{NT}(N=21)$ & $\begin{array}{l}9.92(1.49) \\
8-12\end{array}$ & 100 & $120.8(12.35)$ & Unmedicated & & & \\
\hline \multirow[t]{2}{*}{$\begin{array}{l}\text { South et al. } \\
\text { (2015) }\end{array}$} & Autism $(N=25$ & $\begin{array}{l}22.51(4.5) \\
17-36\end{array}$ & 76 & $\begin{array}{l}108.46 \\
(12.76)\end{array}$ & NR & \multirow[t]{2}{*}{$\begin{array}{l}\text { ASD } \\
(\text { ADOS-2) }\end{array}$} & \multirow[t]{2}{*}{$\mathrm{O}$} & $\begin{array}{l}11 / 29(38 \%) \\
\text { STROBE }\end{array}$ \\
\hline & $\mathrm{NT}(N=45$ & $\begin{array}{l}21.58(2.35) \\
17-36\end{array}$ & 71.1 & $112.42(8.26)$ & NR & & & \\
\hline \multirow[t]{3}{*}{$\begin{array}{l}\text { South et al. } \\
\text { (2017) }\end{array}$} & Autism $(N=40$ & $\begin{array}{l}22.97(6.75) \\
17-52\end{array}$ & 82.5 & NR & NR & \multirow[t]{3}{*}{$\begin{array}{l}\text { ASD } \\
\text { (ADOS-2) }\end{array}$} & $\mathrm{O}$ & $\begin{array}{l}12 / 29(41 \%) \\
\text { STROBE }\end{array}$ \\
\hline & $\begin{array}{l}\mathrm{NT}+\mathrm{ANX} \\
(N=56)\end{array}$ & $\begin{array}{l}21.68(2.76) \\
18-31\end{array}$ & 26.8 & NR & NR & & & \\
\hline & NT $(N=29$ & $\begin{array}{l}21.61(2.19) \\
17-25\end{array}$ & 72.4 & NR & NR & & & \\
\hline $\begin{array}{l}\text { Yoo et al. } \\
\text { (2014) }\end{array}$ & $\begin{array}{l}\text { Autism INT } \\
(n=23)\end{array}$ & $\begin{array}{l}\Gamma 14.04(1.64) \\
12-18\end{array}$ & 91.3 & $99.26(15.37)$ & NR & $\begin{array}{l}\text { PDD-NOS, } \\
\text { Asperger's }\end{array}$ & I & $\begin{array}{l}18 / 32(56 \%) \\
\text { CONSORT }\end{array}$ \\
\hline & $\begin{array}{l}\text { Autism delayec } \\
\text { INT }(n=24)\end{array}$ & $\begin{array}{l}\mathrm{d} 13.54(1.50) \\
12-18\end{array}$ & 95.8 & $\begin{array}{l}100.46 \\
(16.63)\end{array}$ & NR & $\begin{array}{l}\text { disorder, } \\
\text { autistic } \\
\text { disorder } \\
\text { (ADOS, ADI- } \\
\text { R) }\end{array}$ & & \\
\hline
\end{tabular}


Table 1 (continued)

\begin{tabular}{|c|c|c|c|c|c|c|c|c|}
\hline \multirow{2}{*}{$\begin{array}{l}\text { Study refer- } \\
\text { ence }\end{array}$} & \multicolumn{5}{|c|}{ Participant characteristics } & \multirow{2}{*}{$\begin{array}{l}\text { Autism sub- } \\
\text { type (in-study } \\
\text { measures) }\end{array}$} & \multirow[t]{2}{*}{ Study design } & \multirow{2}{*}{$\begin{array}{l}\text { Reporting } \\
\text { score }\end{array}$} \\
\hline & Study groups & $\begin{array}{l}\text { Age in years } \\
\text { Mean }(S D) \\
\text { range }\end{array}$ & Male (\%) & $\begin{array}{l}\text { FSIQ } \\
\text { Mean }(S D)\end{array}$ & $\begin{array}{l}\text { Medication } \\
\text { status }\end{array}$ & & & \\
\hline \multirow{3}{*}{$\begin{array}{l}\text { Zuk- } \\
\text { erman et al. } \\
\text { (2019a) }\end{array}$} & Autism $(N=55$ & $23.56(2.81)$ & $92.7 \%$ & NR & NR & \multirow{3}{*}{$\begin{array}{l}\text { ASD } \\
\text { (AQ screen- } \\
\text { ing) }\end{array}$} & \multirow[t]{3}{*}{$\mathrm{O}$} & \multirow{3}{*}{$\begin{array}{l}20 / 29(69 \%) \\
\text { STROBE }\end{array}$} \\
\hline & $\begin{array}{l}\mathrm{NT}+\text { social pho } \\
\text { bia }(n=31)\end{array}$ & $-25.06(2.62)$ & $83.9 \%$ & NR & NR & & & \\
\hline & $\mathrm{NT}(n=25$ & $24.56(2.18)$ & $84 \%$ & NR & NR & & & \\
\hline \multirow{2}{*}{$\begin{array}{l}\text { Zuk- } \\
\text { erman et al. } \\
\quad(2019 b)\end{array}$} & Autism $(N=55$ & $\begin{array}{l}23.56(2.81) \\
18-34\end{array}$ & $92.7 \%$ & NR & NR & \multirow{2}{*}{$\begin{array}{l}\text { ASD, } \\
\text { Asperger's } \\
\text { syndrome, } \\
\text { PDD } \\
\text { (AQ screen- } \\
\text { ing) }\end{array}$} & \multirow[t]{2}{*}{$\mathrm{O}$} & \multirow[t]{2}{*}{$\begin{array}{l}\text { 17/29 (59\%) } \\
\text { STROBE }\end{array}$} \\
\hline & $\begin{array}{l}\text { Neurotypica } \\
(N=40)\end{array}$ & $\begin{array}{l}125.08(2.67) \\
20-36\end{array}$ & $82.5 \%$ & NR & NR & & & \\
\hline
\end{tabular}

FSIQ full-scale IQ; $N R$ not reported; $S D$ standard deviation

Groups: $A g C C$ agenesis of the corpus callosum; $A L X$ alexithymia; $A N X$ anxiety; Autism participants with a diagnosed autism spectrum disorder; $I N T$ received intervention; $N T$ neurotypical = participants without a diagnosed autism spectrum disorder; $W L C$ wait list control

Autism subtypes: $A S D$ autism spectrum disorder; $P D D(-N O S)$ pervasive developmental disorder (-not otherwise specified)

Measures: $A D I-R$ autism diagnostic interview-revised; $A D O S$ autism diagnostic observational schedule; $A Q$ autism spectrum quotient; $D I S C O$ diagnostic interview for social and communication disorders; $R A A D S-R$ Ritvo Autism Asperger Diagnostic Scale Revised; $S C Q$ social communication questionnaire; SRS Social Responsiveness Scale

Study design: $I$ interventional; $O$ observational

Reporting: CONSORT Consolidated Standards of Reporting Trials; STROBE Strengthening the Reporting of Observational studies in Epidemiology

\section{Participant Characteristics}

In total, 750 individuals on the autism spectrum participated in the included research. The majority $(87.7 \%)$ of participants on the autism spectrum were male, with seven (30.4\%) studies reporting on only male participants. Three studies (Ioannou et al., 2020; Maras \& Bowler, 2012; South et al., 2017) involved participants in gender proportions close to those currently estimated, where around one in four individuals on the autism spectrum is female (Loomes et al., 2017). IQ was reported in 20 (87.0\%) articles. Based upon participant descriptive statistics or study inclusion criteria, no studies that reported IQ included participants on the autism spectrum who had an intellectual disability. There was inconsistent reporting of medication use, with only eight $(34.8 \%)$ studies providing this detail. Six of these articles described participants as unmedicated, and two identified that some participants on the autism spectrum were taking prescription psychotropic, hormone, or steroid medications. Similarly, participant race/ethnicity was reported in only four (17.4\%) articles, with a higher proportion of Caucasian participants (62.5-84.4\%) than other races or ethnicities.

Across studies, the reported age of participants on the autism spectrum ranged from 8 to 56 years old. Eleven $(47.8 \%)$ articles reported on child participants (mean age $<18$ years). Of these, six were conducted by the same research group, and only one occurred outside the US or UK (Yoo et al., 2014; South Korea). Twelve (52.2\%) studies reported on adult participants (mean age $\geq 18$ years). Participant demographics across the studies indicate that no study reported on data from older adults ( $>60$ years).

\section{Measures of Trait Anxiety}

All 23 studies include at least one subjective measure of trait anxiety, as shown in Tables 2 and 3. In all cases, higher scores in these questionnaires depicted higher anxiety levels. Of the 11 studies involving children, the majority (72.7\%) reported on the State Trait Anxiety Inventory for ChildrenTrait subscale (STAIC-T) self-report questionnaire. Similarly, studies measuring trait anxiety scores in adults almost exclusively (91.7\%) used the self-reported State Trait Anxiety Inventory-Trait subscale (STAI-T). The four remaining studies used an array of different subjective measures of trait anxiety. While all studies reported on scores from self-report trait anxiety measures, only two $(8.7 \%)$ included additional parent-report data from the Screen of Child Anxiety-Related Disorders (SCARED-P; Mertens et al., 2017) or Spence Child Anxiety Scale (SCAS-P; Mikita et al., 2015) standardised questionnaires. 


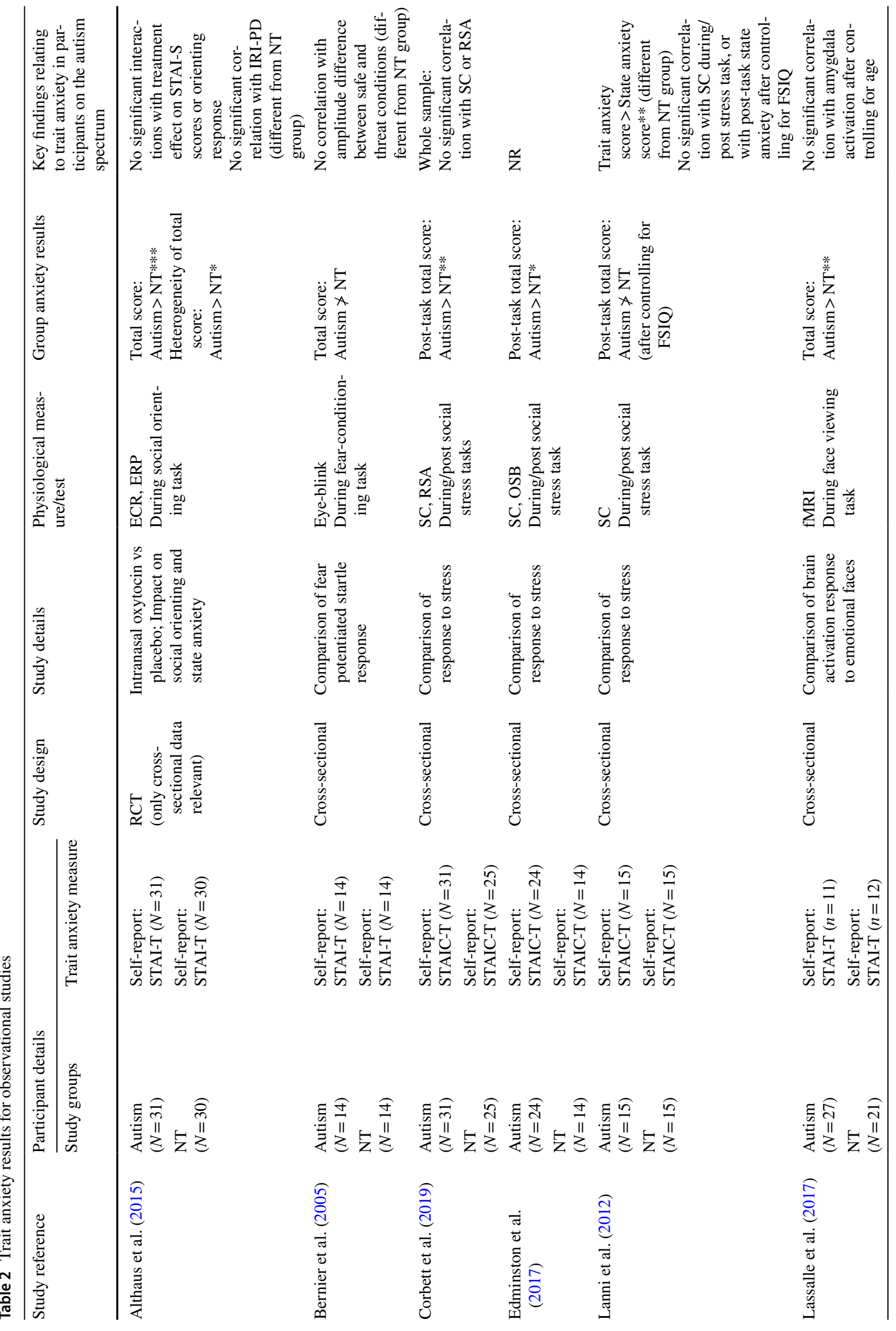




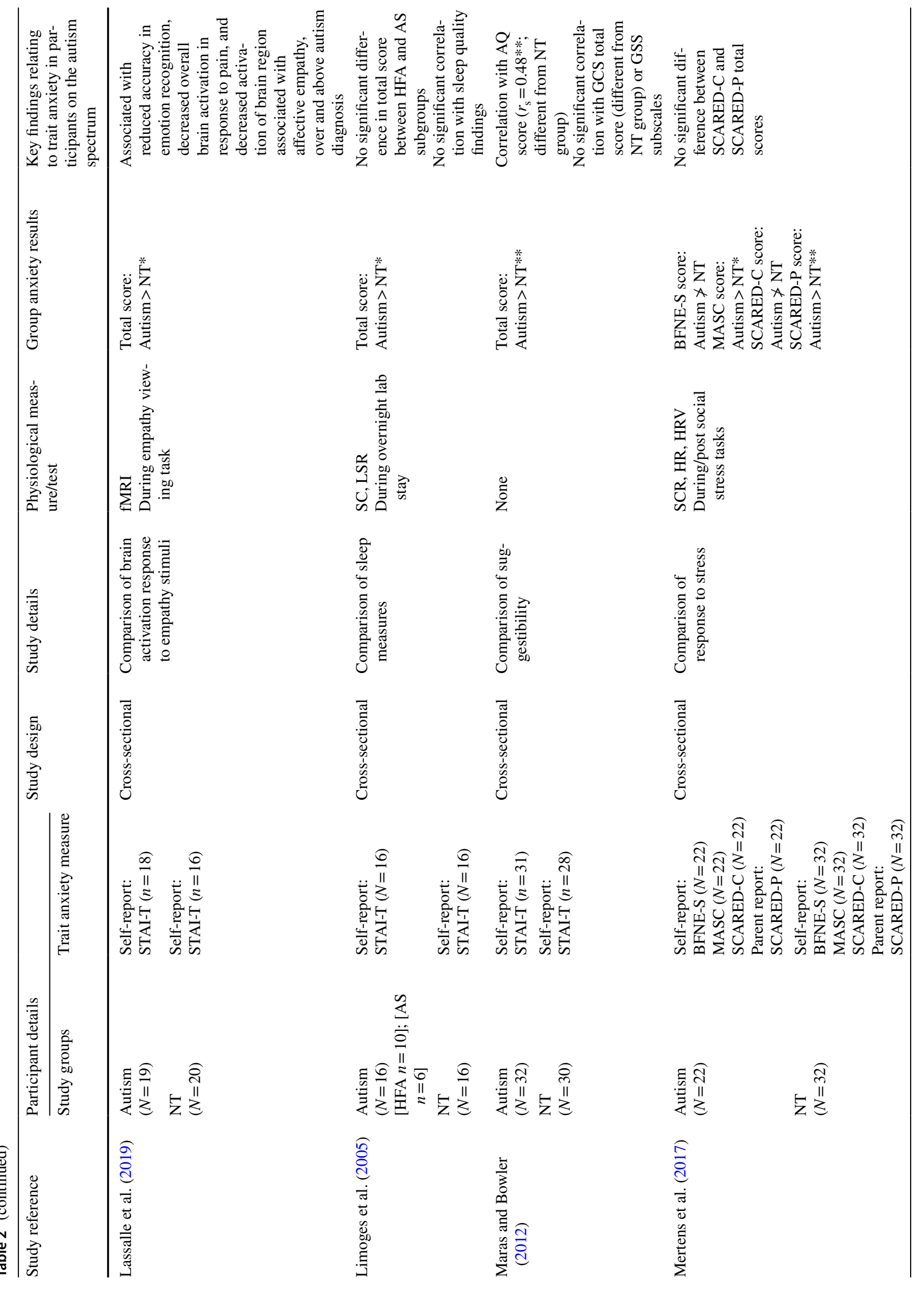




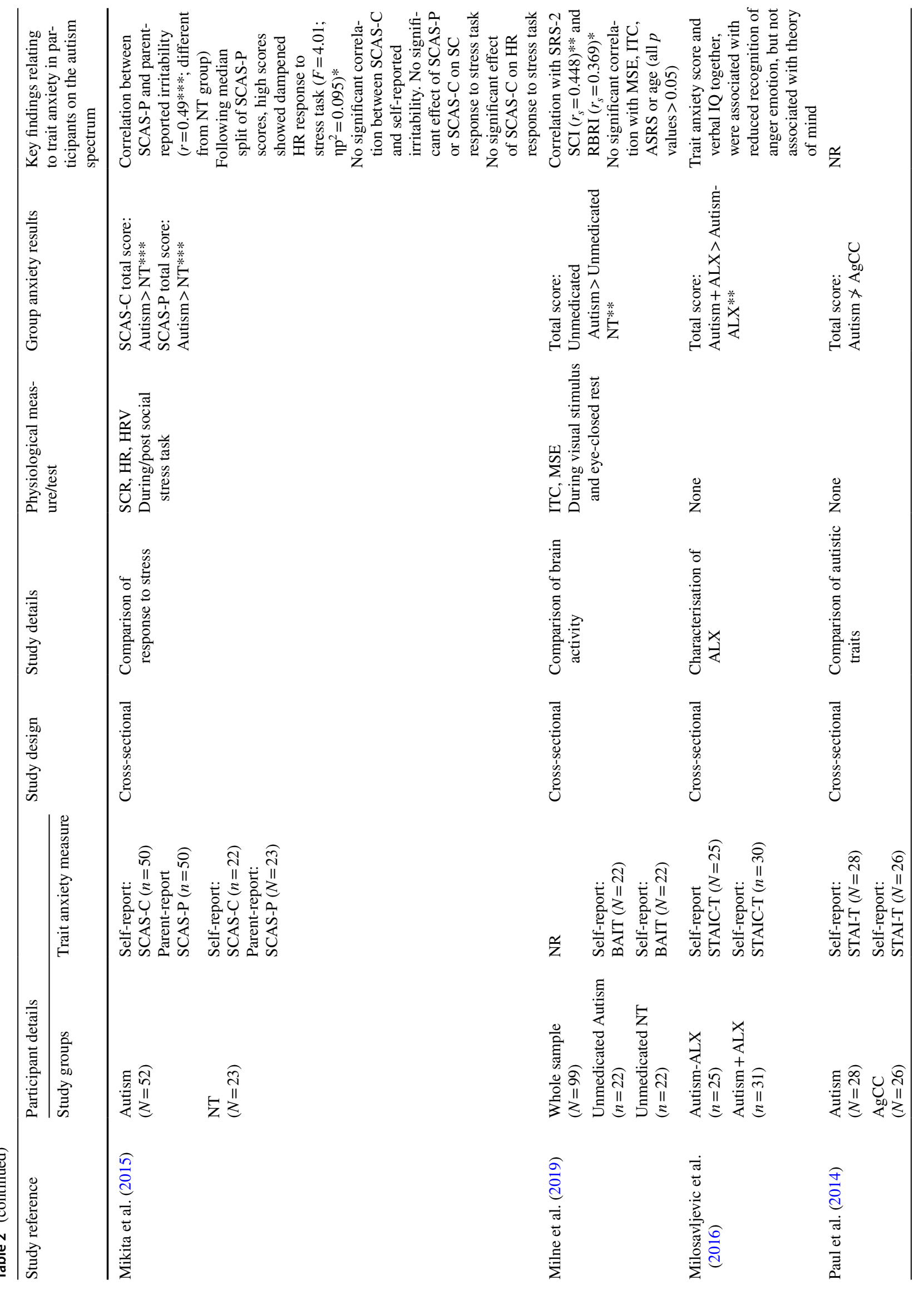




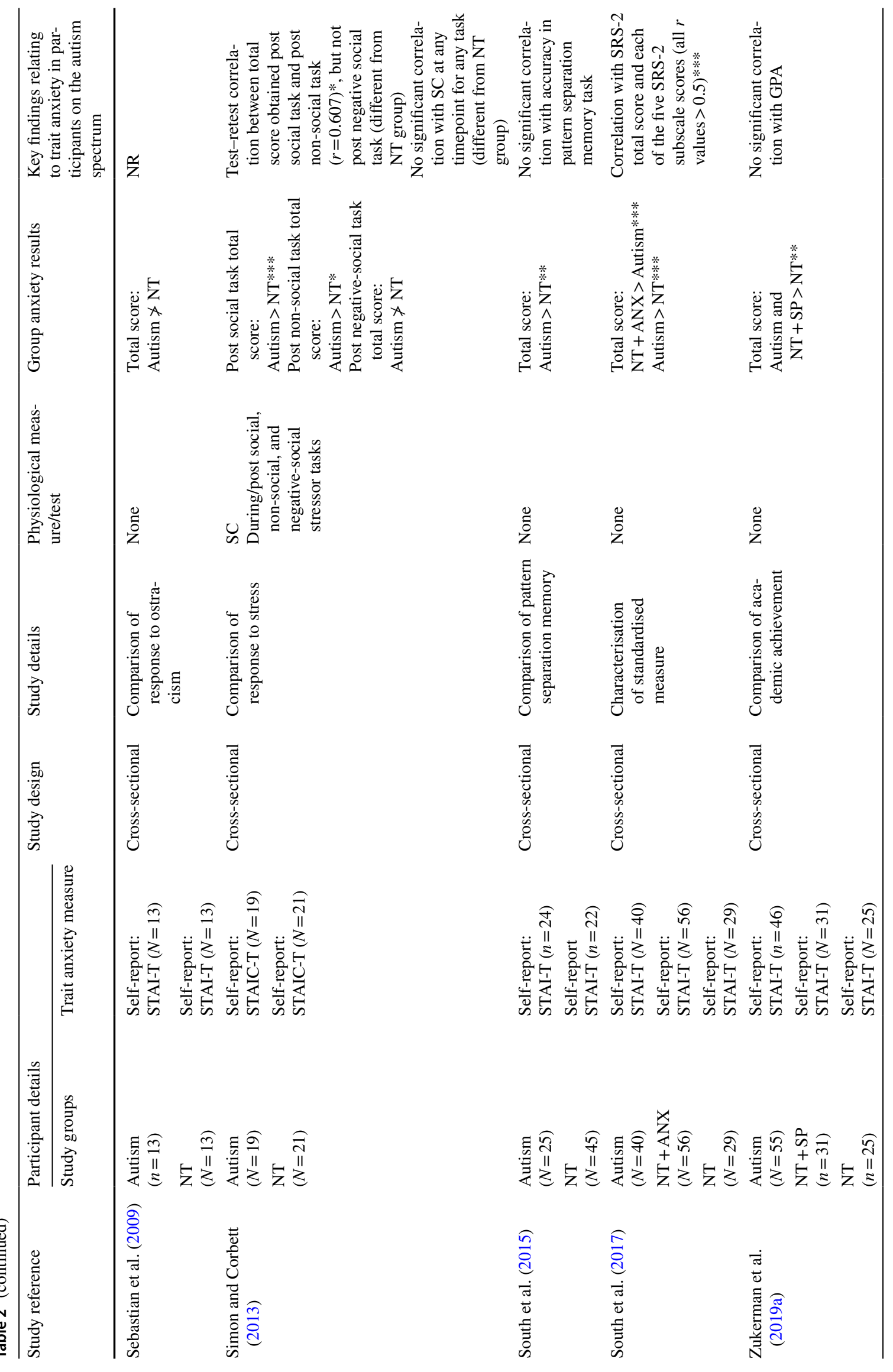




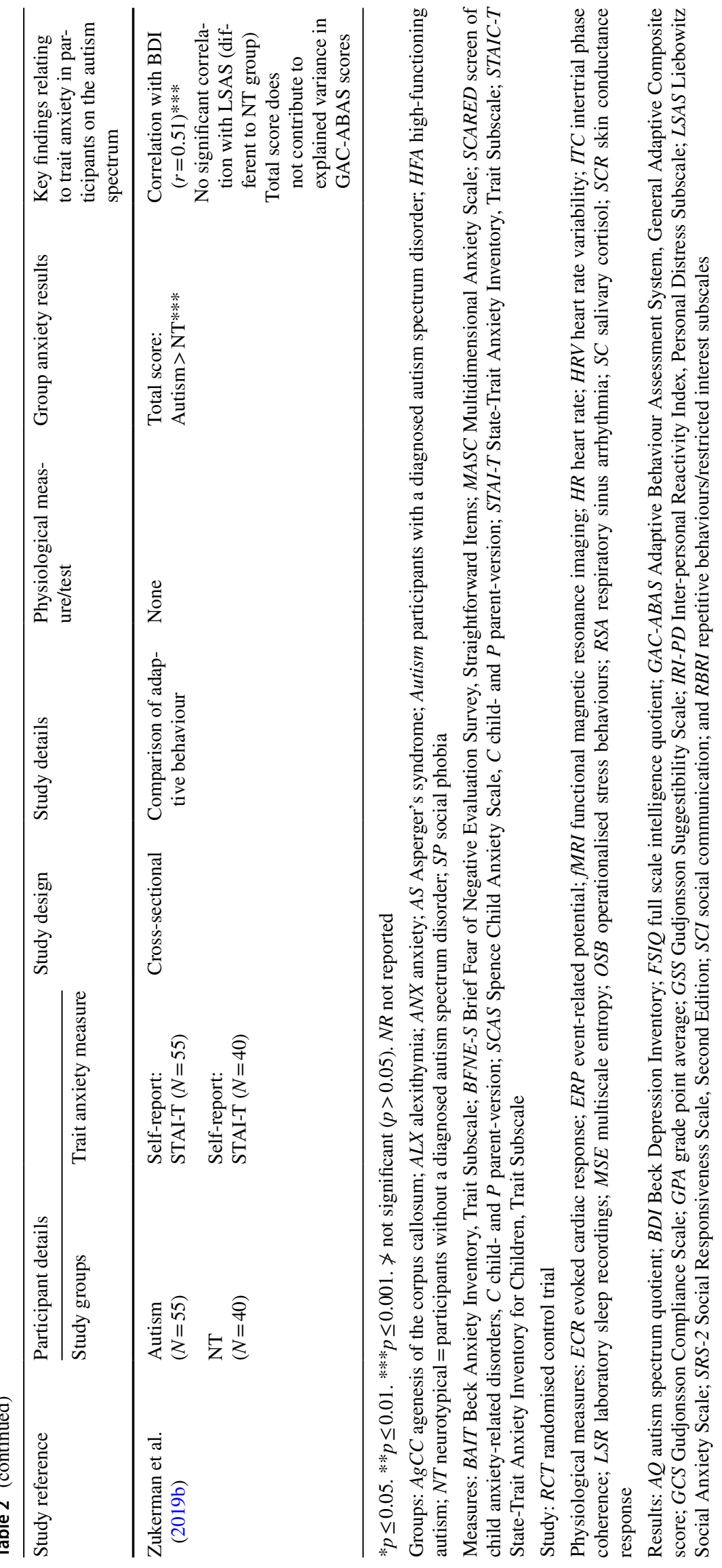




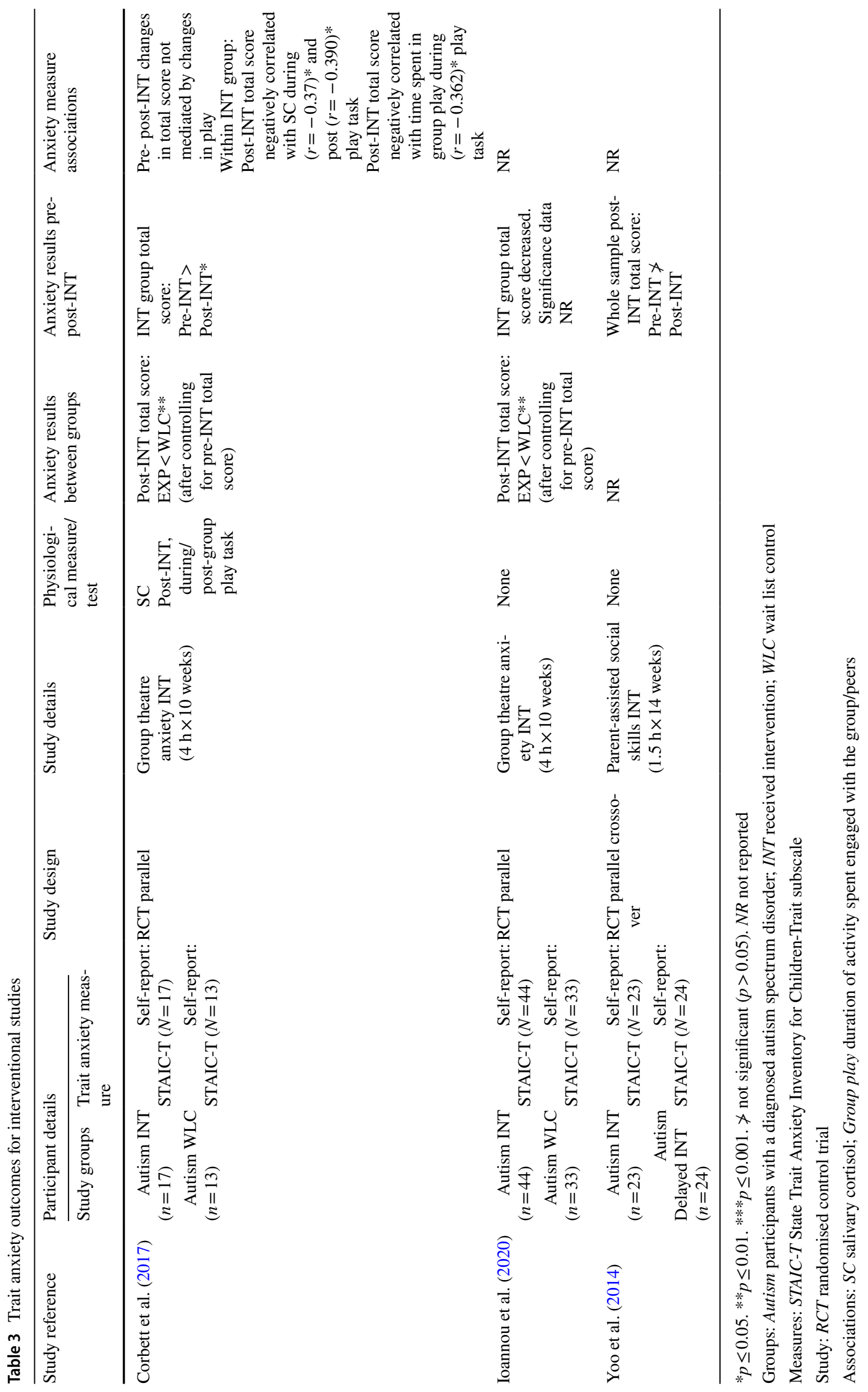




\section{Psychometric Properties of Trait Anxiety Measures}

Few studies reported psychometric properties of the trait anxiety measures used, specifically with reference to participants on the autism spectrum (see Table 4). No studies reported on the validity of the measures used, and only two studies $(8.7 \%)$ reported any reliability data separately for participants on the autism spectrum (Milosavljevic et al., 2016; Yoo et al., 2014). These found that the STAIC-T showed good internal consistency $(\alpha=0.85-0.89)$ in their samples of adolescents (total age range 12-18 years) on the autism spectrum. A further three (13.0\%) studies reported internal consistency of their whole sample, inclusive of neurotypical participants: Corbett et al. (2019) reported $\alpha=0.91$ for the STAIC-T, Milne et al. (2019) reported $\alpha=0.93$ for the Beck Anxiety Inventory, Trait Subscale (BAIT), and Zukerman et al. (2019b) reported $\alpha=0.89$ for the STAI-T. Six (26.1\%) studies reported only the psychometric properties from previously published studies of neurotypical participants, and the remaining 12 (52.2\%) articles described no psychometric properties of the trait anxiety measure used in their study.

\section{Reporting Quality}

Included articles were assessed for quality by determining their adherence to published checklists designed to improve the quality and transparency of reporting (Moher et al., 2010). The global percentage of adequately reported checklist items for each article is provided in Table 1. The 19 objective articles, and the RCT that provided cross-sectional data for this study, were assessed for their adherence to the STROBE statement checklist and reported a mean global score of $52.8 \%(S D=10.23$; range $=35.7-77.4 \%)$ items adequately reported (von Elm et al., 2007). Meanwhile, the remaining three intervention articles were assessed against the CONSORT statement checklist and reported a mean global score of $52.6 \%(\mathrm{SD}=3.2$; range $=50.0$ $56.3 \%$; Schulz et al., 2010). Overall, mean global score was $52.8 \%(S D=10.23)$, and six $(26.1 \%)$ studies adequately reported $<50 \%$ of checklist items.

To assess the areas of reporting strength and weakness among the included studies, the percentage of articles adequately reporting each checklist item was identified. The results of this analysis are included in the Supplementary Materials. Both observation and intervention studies showed a similar pattern, where either most studies reported the detail required for the item, or very few adequately reported

Table 4 Psychometric properties reported, as calculated within the study from autism group participant data

\begin{tabular}{|c|c|c|c|c|c|c|c|c|c|}
\hline \multirow[t]{2}{*}{ Measure name } & \multirow{2}{*}{$\begin{array}{l}\text { Studies } \\
\text { using meas- } \\
\text { ure }(n)\end{array}$} & \multicolumn{3}{|l|}{ Reliability } & \multicolumn{3}{|l|}{ Validity } & \multirow{2}{*}{$\begin{array}{l}\text { In-study data } \\
\text { includes } \\
\text { neurotypical } \\
\text { participants. } \\
\text { Number of stud- } \\
\text { ies (property) }\end{array}$} & \multirow{2}{*}{$\begin{array}{l}\text { Data referenced } \\
\text { from previous } \\
\text { study of neuro- } \\
\text { typical sample. } \\
\text { Number of studies } \\
\text { (property) }\end{array}$} \\
\hline & & $\begin{array}{l}\text { Internal consist- } \\
\text { ency }\end{array}$ & Inter-rater & Test-retest & Criterion & Construct & Content & & \\
\hline STAI-T & 12 & - & - & - & - & - & - & 1 (IC) & $\begin{array}{l}3 \text { (IC), } 1 \text { (TR), } 2 \\
\quad(\mathrm{IC}), \\
2 \text { (Cs) }\end{array}$ \\
\hline STAIC-T & 8 & $\begin{array}{c}2 \\
(\alpha=0.85-0.89)\end{array}$ & - & - & - & - & - & 1 (IC) & 4 (IC) 2 (TR) \\
\hline BAIT & 1 & - & - & - & - & - & - & 1 (IC) & - \\
\hline SCAS-C & 1 & - & - & - & - & - & - & - & - \\
\hline SCAS-P & 1 & - & - & - & - & - & - & - & - \\
\hline BFNE-S & 1 & - & - & - & - & - & - & - & - \\
\hline MASC & 1 & - & - & - & - & - & - & - & 1 (IC) \\
\hline SCARED-C & 1 & - & - & - & - & - & - & - & 1 (IC) \\
\hline SCARED-P & 1 & - & - & - & - & - & - & - & 1 (IC) \\
\hline
\end{tabular}

A dash (-) represents the absence of any included article reporting on a property

Measures: BAIT = Beck Anxiety Inventory, Trait Subscale (Kohn et al. 2008); BFNE-S = Brief Fear of Negative Evaluation Survey, Straightforward Items (Carleton et al. 2011); MASC = Multidimensional Anxiety Scale (March et al. 1997); SCARED = Screen of Child Anxiety Related Disorders, C = Child- and P = Parent-version (Birmaher et al. 1999); SCAS = Spence Child Anxiety Scale, C = Child- and P = Parent-version (Spence 1998); STAI-T = State-Trait Anxiety Inventory, Trait Subscale (Spielberger 1970); STAIC-T State-Trait Anxiety Inventory for Children, Trait Subscale (Spielberger 1973)

Psychometric properties: $\mathrm{Cr}=$ Criterion validity; $\mathrm{Cs}=$ Construct validity; $\mathrm{Ct}=$ Content validity; IC $=$ Internal consistency; IR $=$ Inter-rater reliability; TR $=$ Test-retest reliability 
the item. Specifically, in STROBE assessed studies, of the 31 items applicable to cross-sectional study design, 25 (80.6\%) items were reported either well (by $\geq 75 \%$ of articles) or poorly (by $\leq 25 \%$ of articles; Sorensen et al. 2013). In CONSORT assessed studies, of the 32 items applicable to studies of smaller sample size (rather than large clinical trials), a total of $21(65.6 \%)$ items were reported either well or poorly.

The STROBE and CONSORT checklists have some overlap in items, which allows for the broader picture of quality to be explored. In general, studies were strong at reporting their scientific rationale, objectives, analysis methods, results of statistical analysis (planned and additional), participant descriptive details, interpretation of outcomes, and limitations of the data. In contrast, less than $25 \%$ of studies (regardless of type) detailed the setting of the study, date of study, sample size calculations, analysis results (including estimates and their precision), and details of study funding.

\section{Outcomes}

Outcomes reported in the included studies are extracted and summarised, with analyses relevant to trait anxiety presented in Tables 2 and 3.

\section{Comparison of Trait Anxiety Scores Between Groups}

Overall, 18 (78.3\%) studies included between-group analysis of an autism and a neurotypical (NT) comparison group. Five of the seven studies (71.4\%) that reported on children identified that trait anxiety scores were significantly higher $(p \leq 0.05)$ in the autism group compared to the NT group. In adult participants, 10 of the $11(90.9 \%)$ studies reported significantly higher trait anxiety scores in participants in the autism group compared to the NT group.

Three adult studies compared trait anxiety scores of participants on the autism spectrum with comparison group(s). Two studies (South et al., 2017; Zukerman et al., 2019a) included two comparison groups (1) NT participants with low anxiety, and (2) NT participants with high scores on self-reported general or social anxiety measures. South et al. (2017) reported that the NT+anxiety group scored significantly higher on the STAI-T than both autism and NT groups, and the autism group scored significantly higher than the low anxiety NT group. Zukerman et al. (2019a) found that both the autism group and the NT+ social phobia group had significantly higher trait anxiety scores than the low anxiety NT group. Paul et al. (2014) found that trait anxiety scores were not significantly different in adults on the autism spectrum compared with adults with agenesis of the corpus callosum.

Other between-group differences were reported in several studies. Lanni et al. (2012) identified that children on the autism spectrum (8-12 years old) had significantly higher trait anxiety than state anxiety scores; this pattern was not reported in the children in the NT comparison group. Milosavljevic et al. (2016) reported adolescents (14-17 years old) on the autism spectrum with high alexithymia traits had higher trait anxiety scores than the autism group with low alexithymia. This study also found that participants with alexithymia showed decreased ability to recognise anger emotions compared with those without alexithymia. However, there was no group difference after controlling for STAIC-T and verbal IQ. This finding suggests that trait anxiety scores, in combination with verbal IQ, have a role in the recognition of anger, over and above any effect of alexithymia traits (Milosavljevic et al., 2016). Limoges et al. (2005) reported no significant differences in the trait anxiety scores of participants (16-27 years old) in their high-functioning autism and Asperger's syndrome autism subgroups. Althaus et al. (2015) reported higher heterogeneity in the trait anxiety scores of adults in the autism group compared with the NT group.

There was only one study that compared trait anxiety scores between informants for participants on the autism spectrum. Mertens et al. (2017) reported no significant differences between self- and parent-reported trait anxiety on the SCARED $(p>0.05)$, suggesting that parents of adolescents on the autism spectrum can recognise trait anxiety in their child.

\section{Factors Associated with Trait Anxiety Scores}

A total of $18(78.3 \%)$ studies ran statistical analysis of subjective trait anxiety scores with other study outcomes. Some of these analyses explored the relationship between physiological measures and subjective trait anxiety scores and will be detailed later in the review. Five studies reporting on children evaluated correlations between the STAIC-T and other study outcomes in participants on the autism spectrum, with four of these studies completed by the same research group. Following their theatre-based intervention, Corbett et al. (2017) found that lower STAIC-T scores were correlated with increased time spent in interactive engagement with peers during a play task $(r=-0.362, p=0.05)$. Simon and Corbett (2013) reported a positive test-retest correlation between STAIC-T scores obtained following a social task and a non-social task performed around 6 months apart $(r=0.607, p=0.048)$. Lanni et al. (2012) found no significant correlation between trait anxiety scores and state anxiety scores taken following a stress task.

Ten studies explored correlations between the STAI-T and other outcomes in adulthood. In participants on the autism spectrum, STAI-T scores were found to positively correlate with several subjective measures including the autism spectrum quotient (AQ; rs $=0.48, \mathrm{p}<0.01$; Maras \& Bowler, 2012), Beck depression inventory (BDI; $r=0.51$, 
$p<0.001$; Zukerman et al., 2019b), and the social responsiveness scale (SRS-2) total and subscale scores (all $r>0.5$, $p<0.001$; South et al., 2017). There was also no significant correlation $(p>0.05)$ between STAI-T scores and those of other subjective measures including the inter-personal reactivity index-personal distress scale (IRI-PD; Althaus et al., 2015), Gudjonsson compliance scale (GCS) or Gudjonsson suggestibility subscales (GSS; Maras \& Bowler, 2012), or Liebowitz social anxiety scale (LSAS; Zukerman et al., 2019b). Further, the STAI-T showed no significant correlation with additional study outcomes including sleep quality measures (Limoges et al., 2005), accuracy in pattern separation memory tasks (South et al., 2015), and university academic results (Zukerman et al., 2019a). Finally, trait anxiety scores in adults on the autism spectrum did not contribute to explained variance in measures of adaptive skills (Zukerman et al., 2019b) nor the impact of treatment with oxytocin on state anxiety response following a stress task (Althaus et al., 2015).

The remaining three studies reported results from a variety of trait anxiety measures. Milne et al. (2019) examined correlations between BAIT scores and other study measures in adult participants. BAIT scores were significantly positively correlated with scores on the Adult Repetitive Behaviour Questionnaire (RBRI; $r_{s}=0.369, p<0.05$ ) and the SRS social communicative interaction subscale $\left(r_{s}=0.448\right.$, $p<0.01)$, but not significantly correlated with the ADHD self-report (ASRS) or with age (all $p \mathrm{~s}>0.05$ ). Mikita et al. (2015) used the SCAS self- and parent-reports in child participants to explore correlations between SCAS scores and other study measures. In children on the autism spectrum, they identified that parent-reported anxiety and irritability were positively correlated $(r=0.49, p=0.001)$, but selfreported anxiety and irritability were not $(p>0.05)$.

\section{Physiological Measures Explored Alongside Trait Anxiety Scores}

Six $(26.1 \%)$ studies explored physiological response to stress-inducing tasks, with the aim of evaluating the relationship between these physiological measures and subjective anxiety questionnaires in participants on the autism spectrum. Some of these studies implemented multiple physiological measures: five in total investigated salivary cortisol (four of these were from the same research group), two used electrocardiogram (ECG) to assess various heart rate/rhythm measures, and one examined eye-blink latency and magnitude.

Results correlating trait anxiety scores with salivary cortisol levels were mixed. In children on the autism spectrum, Corbett et al. (2017) reported significant negative correlations between STAIC-T scores and salivary cortisol during $(r=-0.37, p=0.047)$ and following $(r=-0.390$, $p=0.03)$ a social play task after completion of their intervention to reduce trait anxiety scores, whereas crosssectional studies reported no significant correlations during standardised social stress tasks with children (all $p \mathrm{~s}>0.05$; Corbett et al., 2019; Lanni et al., 2012; Mikita et al., 2015; Simon \& Corbett, 2013). Results relating to heart measures were mixed: in adolescents, a dampened heart rate reaction to the stressor was related to high parent-reported anxiety, but not high self-reported anxiety (Mikita et al., 2015), but in children, there was no significant correlation between selfreported trait anxiety scores and respiratory sinus arrythmia (Corbett et al., 2019). In adults, trait anxiety scores showed no significant correlation with eye-blink amplitude differences between safe and threat stressors (Bernier et al., 2005).

A further four (17.4\%) studies measured neural activity in adults through functional magnetic resonance imaging (fMRI) or electroencephalogram (EEG). The two fMRI studies assessed areas of interest to determine relationships between brain activity in these regions and overall scores on subjective trait anxiety measures. Lassalle et al. (2019) reported a between-group comparison where the autism group showed lower accuracy of emotion recognition, decreased whole-brain activation during painful/disgusting stimuli, and decreased activation in brain regions associated with affective empathy; however, there was no group difference after controlling for STAI-T scores. This suggests that trait anxiety scores may be associated with reduced emotion recognition accuracy and decreased empathy for pain, over and above autism diagnostic grouping (Lassalle et al., 2019). Conversely, trait anxiety scores were not associated with change in activation in the amygdala; though authors indicate the low sample number may have affected this result (Lassalle et al., 2017). The EEG studies found no significant correlation between trait anxiety scores and variability of brain activity (Milne et al., 2019) or neural orienting to affective stimuli (Althaus et al., 2015).

\section{Changes in Trait Anxiety Scores Pre- and Post-Intervention}

Four articles in this review report on the outcomes of interventions; however, only three studies reported trait anxiety scores post-intervention so only these three will be discussed here. Corbett et al. (2017) and Ioannou et al. (2020) both reported on RCT parallel design studies evaluating a 10-week group-theatre intervention for anxiety in children on the autism spectrum. Both reported that following intervention, trait anxiety scores in the intervention group were significantly lower than in the wait list control group after controlling for pre-intervention trait anxiety scores (all $p \mathrm{~s} \leq 0.01$ ). Corbett et al. (2017) also reported a significant $(p=0.05)$ decrease in trait anxiety scores in the intervention group from pre- to post-intervention. Yoo et al. (2014) employed an RCT parallel crossover design 
to examine feasibility and treatment efficacy of a 14-week social skills training program in adolescents on the autism spectrum. Trait anxiety scores were measured pre- and postintervention using the STAIC-T, as a secondary outcome of the study. Following completion of the intervention by both the autism intervention and autism delayed-intervention groups, whole sample analysis revealed no significant reduction in STAIC-T scores $(p=0.12)$. In summary, the three intervention studies that report pre- and post-intervention trait anxiety scores suggest that these scores can be reduced with some, but not all, interventions.

\section{Discussion}

This review aimed to gather research that reports on trait anxiety in individuals on the autism spectrum, to outline trends and identify areas for further study. Overall, studies included mainly male participants from Western countries, with no representation of older adults on the autism spectrum or individuals with intellectual disability. Trait anxiety was most commonly measured through a single self-reported subjective questionnaire; however, few studies reported any psychometric properties of their chosen trait anxiety measure for participants on the autism spectrum. Assessment of study reporting using STROBE and CONSORT guidelines identified patterns of strength and weakness in reporting across articles. This review identified that around a quarter of included studies adequately reported $<50 \%$ of checklist items, indicating the need for caution when interpreting study outcomes.

Most studies included both participants on the autism spectrum and a neurotypical comparison group, with the majority reporting significantly higher trait anxiety scores in autism. Comparisons of subjective trait anxiety scores with other study outcomes resulted in mixed findings. Higher trait anxiety scores in participants on the autism spectrum were associated with increased alexithymia and irritability and decreased emotion recognition and social responsiveness. However, many studies found no significant correlation between trait anxiety scores and outcomes including sleep quality, memory, and academic results. Physiological measures like heart rate/rhythm and salivary cortisol showed correlations with trait anxiety scores in some studies but not others, and results relating trait anxiety scores to brain activity were mixed. Finally, intervention studies reported that a theatre-based intervention, but not social skills training intervention, showed significant reductions in trait anxiety score, or lower scores in the experimental group compared to wait list controls following intervention. However, these findings need to be considered with caution given this review found that limited studies have explored the reliability, and no studies have explored the validity, of available trait anxiety measures for individuals on the autism spectrum.

\section{Characteristics of Completed Research}

The majority of included research was undertaken in the USA and the UK, with only three (13.0\%) studies completed outside of North America or Europe. This high proportion of included research from Western countries corresponds with a bibliometric analysis study of autism research output from 2005 to 2014. In this analysis, the USA, the UK, and Canada were the top three countries in terms of research publication output, accounting for $65.4 \%$ of published articles over those 10 years (Sweileh et al., 2016). However, the similarities in the study locations of articles included in this review limit the generalisability of results to other countries, as discussed further below.

Participants on the autism spectrum in the included research were mostly $(87.7 \%)$ male. This equates to less than one in eight participants identifying as female, which falls short of current proportion estimates that one in four individuals on the autism spectrum is female (Loomes et al., 2017). The under-representation of females (and lack of reporting of any participants with non-binary gender) in trait anxiety research limits our understanding of any gender differences that may be present in individuals on the autism spectrum. This is of concern, considering neurotypical research showing differences between males and females in the prevalence (Lago-Mendez et al., 2006; Muris et al., 2001), and impacts (Endler \& Parker, 1990; Meier, 2019; Tan et al., 2011) of trait anxiety. Further research is warranted to explore whether gender-specific experiences of trait anxiety in autism exist and whether they reflect those described in neurotypical literature.

The reported age range of participants across the included studies was 8-56, and within studies that reported IQ data, all participants on the autism spectrum had an IQ $\geq 65$. No study reported the inclusion of older adults ( $>60$ years) or participants with intellectual disability (ID). These absences are consistent with autism research describing the underrepresentation of older adults (Roestorf et al., 2019) and individuals with ID (Russell et al., 2019). However, anxiety levels in autism have been related to both intellectual functioning (van Steensel \& Heeman, 2017) and age (Uljarevic et al., 2020). Having representative samples is important to understand the profile of trait anxiety across a broad range of individuals on the autism spectrum.

\section{Methods Used to Measure Trait Anxiety}

All articles employed a self-report subjective questionnaire to measure trait anxiety scores, of which the most common were the STAIC-T (used in $72.7 \%$ of studies reporting 
on children) and the STAI-T (in $91.7 \%$ of adult and 9.1\% of child studies). These measures are considered the gold standard for measurement of trait anxiety (Coleman et al., 2021; Wong et al., 2018) and are the most commonly used in neurotypical literature (Knowles \& Olatunji, 2020). The use of these well-established measures allows between-group comparison that can draw on existing literature and normative data to inform conclusions. The remaining three studies used one or more other standardised trait anxiety measures (BAIT, BNFE-S, MAST, SCARED, and SCAS), with each measure included in only one study each.

Self-report data were collected in every study. This could be considered a relative strength of this research field, as it contrasts with meta-analysis indicating that parent-report anxiety measures are used more commonly than self-report in autism research (van Steensel \& Heeman, 2017) and relates to assertions that self-report contributes valuable perspectives that may not be recognised by informant reporters (Adams, Clark, et al., 2020; Adams, Simpson, et al., 2020; Keith et al., 2019). Only two employed additional parentreported measures of trait anxiety scores, which highlights a potential area of improvement for this work. Due to differences in anxiety severity rating between informants (Stratis \& Lecavalier, 2015) and differences in anxiety presentation across settings (Adams, Clark, et al., 2019; Adams, Young, et al., 2019), the importance of combining reports from multiple informants in the assessment of anxiety in autism is well-established (Adams Clark, \& Keen, 2019; Adams, Young, et al., 2019; Moss et al., 2015; White, Ollendick, et al., 2009; White, Oswald, et al., 2009). Hence, further research engaging multiple informants and modalities may allow for further insight into the presentation and experience of trait anxiety in individuals on the autism spectrum.

\section{Psychometric Properties of Measures Used}

Only two studies reported any psychometric data for their chosen measure of trait anxiety exclusively for participants on the autism spectrum. Reported psychometric data were limited to calculations of internal consistency, with no studies assessing the validity of the measure used. Additionally, 21 studies included in the current review used measures that have not yet been validated for use in autism populations. Spain et al. (2018) reported similar findings in a systematic review on social anxiety in autism and voiced concern regarding the methodological limitations of using measures without established validity and reliability.

The suitability of using traditional anxiety measures in autism has been questioned (Vasa et al., 2018) due to challenges in anxiety reporting (Hill et al., 2004), diagnostic overshadowing (Kerns \& Kendall, 2012), and the presence of autism-specific anxiety symptoms (Kerns et al., 2014). To address this concern, research has begun to examine the sensitivity, validity, and reliability of traditional anxiety measures in participants on the autism spectrum (Magiati et al., 2017; Park et al., 2020; Stern et al., 2014). However, the consideration of autism-specific presentations or experiences of trait anxiety has not yet been explored, so it is not known if autism-specific measures of trait anxiety are required.

\section{Quality of Research}

No included study reported all required items on the relevant quality checklist, with around a quarter $(26.1 \%)$ of studies adequately reporting less than half of the items recommended. While scientific rationale and study objectives were well-detailed, items relating to the date and location of the study were inadequately reported. This aligns with research identifying that the lack of consideration of external validity in study reporting is a frequent criticism among clinicians (Rothwell, 2005). Details of research location and setting are necessary to confidently judge the generalisability of results. Large-scale factors, such as cultural opinions and national health programs, can significantly affect the diagnostic services accessed by individuals considering an autism diagnosis across locations, potentially influencing the demographics of study participants (Bernier et al., 2010). Inadequate reporting of these items limits the ability of this review to make generalisations regarding trait anxiety in autism. Similarly, the tendency of studies to poorly report the precision of outcome estimates limits the confidence with which results can be interpreted and built upon in future research.

\section{Findings of Completed Research}

Among the 18 studies that ran between-group analyses, most (83.3\%) identified higher scores on trait anxiety measures in participants on the autism spectrum compared with neurotypical peers. This outcome is unsurprising given the common reporting of elevated anxiety in autism (Hollocks et al., 2019; van Steensel et al., 2011). To best interpret the finding of elevated trait anxiety scores in autism, it is important to consider anxiety severity as a continuum across normal and abnormal psychology. This is demonstrated in individuals on the autism spectrum, where the prevalence of anxiety disorders is around $40 \%$, whereas impairing subclinical anxiety symptoms are considerably higher (70-88.9\%; Adams, Clark, et al., 2019; Adams, Young, et al., 2019; Kaat et al., 2013). Higher levels of trait anxiety indicate a tendency towards anxious response. This may manifest as higher anxiety across a variety of settings but may be expressed through symptoms which may not reach clinical thresholds for any 
specific anxiety disorder. In this way, measurement of trait anxiety may allow exploration of those subclinical, but still impairing, levels of anxiety identified in autism.

Studies explored the relationship between trait anxiety scores and a diverse range of outcomes. Three (13.0\%) adult studies found positive correlations between trait anxiety scores and measures commonly used to assess characteristics associated with autism (the AQ and SRS-2). Whilst this might suggest higher trait anxiety scores in those with more autism characteristics, there may also be an issue of measurement; i.e. difficulties in discrimination may be due to the overlap between anxiety symptoms and core autism characteristics (Kerns \& Kendall, 2012). For example, the AQ and SRS-2 show poor ability to discriminate between anxiety and autism (Capriola-Hall et al., 2021; Cath et al., 2008). These findings therefore reiterate the need for careful consideration when attempting differential diagnoses.

Two $(8.7 \%)$ studies indicated a relationship between higher trait anxiety scores and poorer emotion recognition. This result is consistent with neurotypical literature in which one study reported a negative correlation between trait anxiety scores and emotion recognition (Kessler et al., 2007), and meta-analysis found a significant impairment in emotion recognition in adults with anxiety disorders (Demenescu et al., 2010). The underlying mechanism for this impairment is unknown; however, attentional biases have been implicated, whereby reduced accuracy in the recognition of angry faces is due to the attentional avoidance of these faces. This avoidance is a form of cognitive processing (Cisler \& Koster, 2010) that has been associated with emotion regulation goals in neurotypical trait anxiety (Koster et al., 2006) and presents an intriguing avenue for further research in autism.

Studies assessing physiological measures alongside trait anxiety scores reported mixed results. In one study, high parent-reported trait anxiety scores predicted dampened heart rate reactivity to a high-stress task. This result is consistent with the findings of research involving adolescents on the autism spectrum with co-occurring anxiety disorders, supporting the suggestion that individuals with habitually high anxiety become desensitised to stress tasks (Hollocks et al., 2016). However, a second study included in this review reported no significant correlation between trait anxiety scores and respiratory sinus arrhythmia. Mixed results were also found among the five studies that assessed salivary cortisol response to stress, where one indicated a negative correlation with trait anxiety scores, but the rest found no significant correlation. These conflicting results highlight the need for further research exploring the mediating effect of trait anxiety on physiological stress response in autism.

Mixed results were reported in the intervention studies. Two studies investigating peer-mediated, group-theatrebased interventions indicated success in reducing trait anxiety scores compared with a wait list control group; however, a third study using a parent-assisted social skills training program saw no significant change. Cultural influence on anxiety may have impacted study outcomes, since Yoo et al. (2014) involved participants on the autism spectrum exclusively from South Korea, whereas Corbett et al. (2017) and Ioannou et al. (2020) studies were both undertaken in the UK. Hofmann and Hinton (2014) outlined several cultural influences that can lead to profound differences in the presentation of anxiety across cultures. Although Yoo et al. (2014) used a Korean-adjusted intervention program and several subjective measures that had been validated in Korean populations, the measure of trait anxiety used had not been adjusted from the original, potentially impacting the reporting of trait anxiety scores in this population. Given the considerable number of intervention studies targeted at reducing anxiety disorders across populations on the autism spectrum (Delli et al., 2018), it is surprising that only three studies assessed the impact of an intervention on trait anxiety. This highlights the need for research exploring both interventions to reduce trait anxiety and measures sensitive enough to identify these changes.

\section{Limitations}

A systematic search of eight electronic databases was conducted, which included a proximity search for key terms "trait" and "anxiety". Articles were then included if the author indicated that trait anxiety was an outcome being measured. It is possible that measures of trait anxiety without these key terms in their title (e.g. Revised Child Anxiety and Depression Scale) may not have been captured in database searches. Further, the intent of the study to investigate trait anxiety may have been outlined in the body of the article and therefore missed in database searches which targeted title and abstract only.

To reduce risk of bias associated with studies of a small sample size, single case design and case studies were excluded from the review. However, single case design can provide an experimentally rigorous framework that is often preferred by researchers with a particular interest in providing an evidence base for individualised interventions within special education settings (Maggin et al., 2021). The selection criteria of the current review may have led to the exclusion of trait anxiety research in these areas of interest, so future reviews may wish to include studies with such designs.

The characteristics, methods, quality, and outcomes of 23 studies were analysed in this review. Findings revealed a narrow representation of individuals on the autism spectrum as participants were mainly males from Western countries, reducing the generalisability of study findings across the broader autism population. Similarly, inadequate study 
reporting, and the use of outcome measures not yet validated within individuals on the autism spectrum, indicates that study outcomes should be considered with caution.

\section{Implications for Future Research}

Areas of consideration for future research include the evaluation of psychometric properties of existing trait anxiety measures across populations of individuals on the autism spectrum. Without these data, the validity of research outcomes to date is questionable, and valid analysis of future intervention trials is not possible. Additionally, research involving more diverse participant samples would improve the generalisability of trait anxiety results to all individuals on the autism spectrum. Finally, increasing the transparency of reporting would allow risk of bias analysis, improving confidence in the outcomes being reported.

Supplementary Information The online version contains supplementary material available at https://doi.org/10.1007/s40489-022-00308-8.

Author Contribution Rebecca Jolliffe designed the study, wrote the protocol, conducted literature searches and the screening of the articles, extracted data, assessed study quality, synthesised the results, and contributed to the discussion. Dawn Adams designed the study, assessed study quality, synthesised results, and contributed to the discussion. Kate Simpson designed the study, synthesised results, and contributed to the discussion. All authors contributed to and have approved the final manuscript.

Funding Open Access funding enabled and organized by CAUL and its Member Institutions. The authors acknowledge the financial support of the Australian Research Council, the Commonwealth Government's Research Training Program, and Griffith University. The funding contributors had no role in the study design, collection, analysis, or interpretation of the data, writing the manuscript, or the decision to submit the paper for publication. The views expressed are those of the authors and not necessarily those of any funding bodies.

\section{Declarations}

Conflict of Interest The authors declare no competing interests.

Open Access This article is licensed under a Creative Commons Attribution 4.0 International License, which permits use, sharing, adaptation, distribution and reproduction in any medium or format, as long as you give appropriate credit to the original author(s) and the source, provide a link to the Creative Commons licence, and indicate if changes were made. The images or other third party material in this article are included in the article's Creative Commons licence, unless indicated otherwise in a credit line to the material. If material is not included in the article's Creative Commons licence and your intended use is not permitted by statutory regulation or exceeds the permitted use, you will need to obtain permission directly from the copyright holder. To view a copy of this licence, visit http://creativecommons.org/licenses/by/4.0/.

\section{References}

Adams, D., Clark, M., \& Keen, D. (2019a). Using self-report to explore the relationship between anxiety and quality of life in children on the autism spectrum. Autism Research, 12(10), 1505-1515. https://doi.org/10.1002/aur.2155

Adams, D., Clark, M., \& Simpson, K. (2020a). The relationship between child anxiety and the quality of life of children, and parents of children, on the autism spectrum. Journal of Autism and Developmental Disorders, 50(5), 1756-1769. https://doi.org/10. 1007/s10803-019-03932-2

Adams, D., Simpson, K., \& Keen, D. (2020b). Exploring anxiety at home, school, and in the community through self-report from children on the autism spectrum. Autism Research, 13(4), 603-614. https://doi.org/10.1002/aur.2246

Adams, D., Young, K., Simpson, K., \& Keen, D. (2019b). Parent descriptions of the presentation and management of anxiousness in children on the autism spectrum. Autism, 23(4), 980-992. https://doi.org/10.1177/1362361318794031

Althaus, M., Groen, Y., Wijers, A., Noltes, H., Tucha, O., \& Hoekstra, P. (2015). Oxytocin enhances orienting to social information in a selective group of high-functioning male adults with autism spectrum disorder. Neuropsychologia, 79(Part A), 53-69. https:// doi.org/10.1016/j.neuropsychologia.2015.10.025

American Psychiatric Association. (1994). DSM-IV: Diagnostic and statistical manual of mental disorders, 4th ed. American Psychiatric Association.

American Psychiatric Association. (2013). Diagnostic and statistical manual of mental disorders: DSM-5 (5th ed.). American Psychiatric Association.

Bernier, R., Dawson, G., Panagiotides, H., \& Webb, S. (2005). Individuals with autism spectrum disorder show normal responses to a fear potential startle paradigm. Journal of Autism and Developmental Disorders, 35(5), 575-583. https://doi.org/10.1007/ s10803-005-0002-0

Bernier, R., Mao, A., \& Yen, J. (2010). Psychopathology, families, and culture: Autism. Child and Adolescent Psychiatric Clinics of North America, 19(4), 855-867. https://doi.org/10.1016/j.chc. 2010.07.005

Birmaher, B., Brent, D. A., Chiappetta, L., Bridge, J., Monga, S., \& Baugher, M. (1999). Psychometric properties of the screen for child anxiety related emotional disorders (SCARED): A replication study. Journal of the American Academy of Child and Adolescent Psychiatry, 38(10), 1230-1236. https://doi.org/10.1097/ 00004583-199910000-00011

Capriola-Hall, N. N., McFayden, T., Ollendick, T. H., \& White, S. W. (2021). Caution when screening for autism among socially anxious youth. Journal of Autism and Developmental Disorders, 51(5), 1540-1549. https://doi.org/10.1007/s10803-020-04642-w

Carleton, R. N., Collimore, K. C., McCabe, R. E., \& Antony, M. M. (2011). Addressing revisions to the brief fear of negative evaluation scale: Measuring fear of negative evaluation across anxiety and mood disorders. Journal of Anxiety Disorders, 25(6), 822828. https://doi.org/10.1016/j.janxdis.2011.04.002

Cath, D. C., Ran, N., Smit, J. H., van Balkom, A. J., \& Comijs, H. C. (2008). Symptom overlap between autism spectrum disorder, generalized social anxiety disorder and obsessive-compulsive disorder in adults: A preliminary case-controlled study. Psychopathology, 4l(2), 101-110. https://doi.org/10.1159/000111555

Cisler, J. M., \& Koster, E. H. (2010). Mechanisms of attentional biases towards threat in anxiety disorders: An integrative review. Clinical Psychology Review, 30(2), 203-216. https://doi.org/10.1016/j. cpr.2009.11.003

Coleman, K. D., Chow, Y., Jacobson, A., Hainsworth, K. R., \& Drendel, A. L. (2021). An evaluation of short anxiety measures for use 
in the emergency department. The American Journal of Emergency Medicine, 50, 679-682. https://doi.org/10.1016/j.ajem. 2021.09.028

Corbett, B. A., Blain, S. D., Ioannou, S., \& Balser, M. (2017). Changes in anxiety following a randomized control trial of a theatre-based intervention for youth with autism spectrum disorder. Autism: The International Journal of Research and Practice, 21(3), 333-343. https://doi.org/10.1177/1362361316643623

Corbett, B. A., Muscatello, R. A., \& Baldinger, C. (2019). Comparing stress and arousal systems in response to different social contexts in children with ASD. Biological Psychology, 140, 119-130. https://doi.org/10.1016/j.biopsycho.2018.12.010

Delli, C. K. S., Polychronopoulou, S. A., Kolaitis, G. A., \& Antoniou, A. G. (2018). Review of interventions for the management of anxiety symptoms in children with ASD. Neuroscience and Biobehavioral Reviews, 95, 449-463. https://doi.org/10.1016/j. neubiorev.2018.10.023

Demenescu, L. R., Kortekaas, R., den Boer, J. A., \& Aleman, A. (2010). Impaired attribution of emotion to facial expressions in anxiety and major depression. PLOS ONE, 5(12), e15058. https://doi.org/10.1371/journal.pone.0015058

Edmiston, E., Blain, S. D., \& Corbett, B. A. (2017). Salivary cortisol and behavioral response to social evaluative threat in adolescents with autism spectrum disorder. Autism Research, 10(2), 346-358. https://doi.org/10.1002/aur.1660

Endler, N. S., \& Parker, J. D. A. (1990). State and trait anxiety, depression and coping styles. Australian Journal of Psychology, 42(2), 207-220. https://doi.org/10.1080/00049539008260119

Hill, E. L., Berthoz, S., \& Frith, U. (2004). Brief report: Cognitive processing of own emotions in individuals with autistic spectrum disorder and in their relatives. Journal of Autism and Developmental Disorders, 34(2), 229-235. https://doi.org/10. 1023/B:JADD.0000022613.41399.14

Hofmann, S. G., \& Hinton, D. E. (2014). Cross-cultural aspects of anxiety disorders. Current Psychiatry Reports, 16(6), 450. https://doi.org/10.1007/s11920-014-0450-3

Hollocks, M. J., Lerh, J. W., Magiati, I., Meiser-Stedman, R., \& Brugha, T. S. (2019). Anxiety and depression in adults with autism spectrum disorder: A systematic review and meta-analysis. Psychological Medicine, 49(4), 559-572. https://doi.org/ 10.1017/S0033291718002283

Hollocks, M. J., Pickles, A., Howlin, P., \& Simonoff, E. (2016). Dual cognitive and biological correlates of anxiety in autism spectrum disorders. Journal of Autism and Developmental Disorders, 46(10), 3295-3307. https://doi.org/10.1007/ s10803-016-2878-2

Houston, B. K., Fox, J. E., \& Forbes, L. (1984). Trait anxiety and children's state anxiety, cognitive behaviors, and performance under stress. Cognitive Therapy and Research, 8(6), 631-641. https://doi.org/10.1007/BF01173261

Ioannou, S., Key, A. P., Muscatello, R. A., Klemencic, M., \& Corbett, B. A. (2020). Peer actors and theater techniques play pivotal roles in improving social play and anxiety for children with autism. Frontiers in Psychology, 11, 908. https://doi.org/10.3389/fpsyg. 2020.00908

Jonsson, P. (2007). Respiratory sinus arrhythmia as a function of state anxiety in healthy individuals. International Journal of Psychophysiology, 63(1), 48-54. https://doi.org/10.1016/j.ijpsycho.2006. 08.002

Kaat, A. J., Gadow, K. D., \& Lecavalier, L. (2013). Psychiatric symptom impairment in children with autism spectrum disorders. Journal of Abnormal Child Psychology, 41(6), 959-969. https://doi. org/10.1007/s10802-013-9739-7

Kanne, S. M., Abbacchi, A. M., \& Constantino, J. N. (2009). Multiinformant ratings of psychiatric symptom severity in children with autism spectrum disorders: The importance of environmental context. Journal of Autism and Developmental Disorders, 39(6), 856-864. https://doi.org/10.1007/s10803-009-0694-7

Keith, J. M., Jamieson, J. P., \& Bennetto, L. (2019). The importance of adolescent self-report in autism spectrum disorder: Integration of questionnaire and autonomic measures. Journal of Abnormal Child Psychology, 47(4), 741-754. https://doi.org/10.1007/ s10802-018-0455-1

Kerns, C. M., \& Kendall, P. C. (2012). The presentation and classification of anxiety in autism spectrum disorder. Clinical Psychology: Science and Practice, 19(4), 323-347. https://doi.org/10.1111/ cpsp. 12009

Kerns, C. M., Kendall, P. C., Berry, L., Souders, M. C., Franklin, M. E., Schultz, R. T., Miller, J., \& Herrington, J. (2014). Traditional and atypical presentations of anxiety in youth with autism spectrum disorder. Journal of Autism and Developmental Disorders, 44(11), 2851-2861. https://doi.org/10.1007/s10803-014-2141-7

Kessler, H., Roth, J., von Wietersheim, J., Deighton, R. M., \& Traue, H. C. (2007). Emotion recognition patterns in patients with panic disorder. Depression and Anxiety, 24(3), 223-226. https://doi.org/ $10.1002 /$ da.20223

Knowles, K. A., \& Olatunji, B. O. (2020). Specificity of trait anxiety in anxiety and depression: Meta-analysis of the state-trait anxiety inventory. Clinical Psychology Review, 82, 101928. https://doi. org/10.1016/j.cpr.2020.101928

Kohn, P. M., Kantor, L., DeCicco, T. L., \& Beck, A. T. (2008). The beck anxiety inventory-trait (BAIT): A measure of dispositional anxiety not contaminated by dispositional depression. Journal of Personality Assessment, 90(5), 499-506. https://doi.org/10.1080/ 00223890802248844

Koster, E. H., Crombez, G., Verschuere, B., Van Damme, S., \& Wiersema, J. R. (2006). Components of attentional bias to threat in high trait anxiety: Facilitated engagement, impaired disengagement, and attentional avoidance. Behaviour Research and Therapy, 44(12), 1757-1771. https://doi.org/10.1016/j.brat.2005.12. 011

Lago-Mendez, L., Diniz-Freitas, M., Senra-Rivera, C., Seoane-Pesqueira, G., Gandara-Rey, J. M., \& Garcia-Garcia, A. (2006). Dental anxiety before removal of a third molar and association with general trait anxiety. Journal of Oral and Maxillofacial Surgery, 64(9), 1404-1408. https://doi.org/10.1016/j.joms.2006.05.030

Lanni, K. E., Schupp, C. W., Simon, D., \& Corbett, B. A. (2012). Verbal ability, social stress, and anxiety in children with autistic disorder. Autism, 16(2), 123-138. https://doi.org/10.1177/13623 61311425916

Lassalle, A., Johnels, J. A., Zurcher, N. R., Hippolyte, L., Billstedt, E., Ward, N., Lemonnier, E., Gillberg, C., \& Hadjikhani, N. (2017). Hypersensitivity to low intensity fearful faces in autism when fixation is constrained to the eyes. Human Brain Mapping, 38(12), 5943-5957. https://doi.org/10.1167/17.10.833

Lassalle, A., Zurcher, N. R., Porro, C. A., Benuzzi, F., Hippolyte, L., Lemonnier, E., Asberg Johnels, J., \& Hadjikhani, N. (2019). Influence of anxiety and alexithymia on brain activations associated with the perception of others' pain in autism. Social Neuroscience, 14(3), 359-377. https://doi.org/10.1080/17470919.2018.1468358

Lau, J. Y., Eley, T. C., \& Stevenson, J. (2006). Examining the statetrait anxiety relationship: A behavioural genetic approach. Journal of Abnormal Child Psychology, 34(1), 19-27. https://doi.org/10. 1007/s10802-005-9006-7

Limoges, É., Mottron, L., Bolduc, C., Berthiaume, C., \& Godbout, R. (2005). Atypical sleep architecture and the autism phenotype. Brain, 128(5), 1049-1061. https://doi.org/10.1093/brain/awh425

Loomes, R. D., Hull, L. M., \& Mandy, W. P. L. D. P. (2017). What is the male-to-female ratio in autism spectrum disorder? A systematic review and meta-analysis. Journal of the American Academy of Child and Adolescent Psychiatry, 56(6), 466-474. https://doi. org/10.1016/j.jaac.2017.03.013 
Maggin, D. M., Barton, E., Reichow, B., Lane, K., \& Shogren, K. A. (2021). Commentary on the what works clearinghouse standards and procedures handbook (v. 4.1) for the review of single-case research. Remedial and Special Education. https://doi.org/10. 1177/07419325211051317

Magiati, I., Lerh, J. W., Hollocks, M. J., Uljarevic, M., Rodgers, J., McConachie, H., Ozsivadjian, A., South, M., Van Hecke, A., Hardan, A., Libove, R., Leekam, S., \& Simonoff, E. (2017). The measurement properties of the spence children's anxiety scaleparent version in a large international pooled sample of young people with autism spectrum disorder. Autism Research, 10(10), 1629-1652. https://doi.org/10.1002/aur.1809

Maras, K. L., \& Bowler, D. M. (2012). Brief report: Suggestibility, compliance and psychological traits in high-functioning adults with autism spectrum disorder. Research in Autism Spectrum Disorders, 6(3), 1168-1175. https://doi.org/10.1016/j.rasd.2012. 03.013

March, J. S., Parker, J. D., Sullivan, K., Stallings, P., \& Conners, C. K. (1997). The multidimensional anxiety scale for children (MASC): Factor structure, reliability, and validity. Journal of the American Academy of Child and Adolescent Psychiatry, 36(4), 554-565. https://doi.org/10.1097/00004583-199704000-00019

Mazzone, L., Ruta, L., \& Reale, L. (2012). Psychiatric comorbidities in asperger syndrome and high functioning autism: Diagnostic challenges. Annals of General Psychiatry, 11(16), 1-13. https:// doi.org/10.1186/1744-859X-11-16

McEwen, B. S., \& Gianaros, P. J. (2011). Stress- and allostasis-induced brain plasticity. Annual Review of Medicine, 62(1), 431-445. https://doi.org/10.1146/annurev-med-052209-100430

McHugh, M. L. (2012). Interrater reliability: The kappa statistic. Biochemia Medica, 22(3), 276-282. https://doi.org/10.11613/BM. 2012.031

Meier, S. T. (2019). Elevated trait anxiety: Obstacle to progress with female clients? Professional Psychology: Research and Practice, 50(1), 33-38. https://doi.org/10.1037/pro0000211

Mertens, J., Zane, E. R., Neumeyer, K., \& Grossman, R. B. (2017). How anxious do you think I am? Relationship between state and trait anxiety in children with and without ASD during social tasks. Journal of Autism and Developmental Disorders, 47(12), 36923703. https://doi.org/10.1007/s10803-016-2979-y

Mikita, N., Hollocks, M. J., Papadopoulos, A. S., Aslani, A., Harrison, S., Leibenluft, E., Simonoff, E., \& Stringaris, A. (2015). Irritability in boys with autism spectrum disorders: An investigation of physiological reactivity. Journal of Child Psychology and Psychiatry, 56(10), 1118-1126. https://doi.org/10.1111/jcpp.12382

Milne, E., Gomez, R., Giannadou, A., \& Jones, M. (2019). Atypical EEG in autism spectrum disorder: Comparing a dimensional and a categorical approach. Journal of Abnormal Psychology, 128(5), 442-452. https://doi.org/10.1037/abn0000436

Milosavljevic, B., Carter Leno, V., Simonoff, E., Baird, G., Pickles, A., Jones, C. R., Erskine, C., Charman, T., \& Happe, F. (2016). Alexithymia in adolescents with autism spectrum disorder: Its relationship to internalising difficulties, sensory modulation and social cognition. Journal of Autism and Developmental Disorders, 46(4), 1354-1367. https://doi.org/10.1007/s10803-015-2670-8

Moher, D., Hopewell, S., Schulz, K. F., Montori, V., Gotzsche, P. C., Devereaux, P. J., Elbourne, D., Egger, M., \& Altman, D. G. (2010). CONSORT 2010 explanation and elaboration: Updated guidelines for reporting parallel group randomised trials. $B M J$, 340, c869. https://doi.org/10.1136/bmj.c869

Moss, P., Howlin, P., Savage, S., Bolton, P., \& Rutter, M. (2015). Self and informant reports of mental health difficulties among adults with autism findings from a long-term follow-up study. Autism, 19(7), 832-841. https://doi.org/10.1177/1362361315585916

Muris, P., Schmidt, H., Merckelbach, H., \& Schouten, E. (2001). Anxiety sensitivity in adolescents: Factor structure and relationships to trait anxiety and symptoms of anxiety disorders and depression. Behaviour Research and Therapy, 39(1), 89-100. https://doi.org/ 10.1016/s0005-7967(99)00179-5

Page, M. J., McKenzie, J. E., Bossuyt, P. M., Boutron, I., Hoffmann, T. C., Mulrow, C. D., Shamseer, L., Tetzlaff, J. M., Akl, E. A., Brennan, S. E., Chou, R., Glanville, J., Grimshaw, J. M., Hrobjartsson, A., Lalu, M. M., Li, T., Loder, E. W., Mayo-Wilson, E., McDonald, S., ... Moher, D. (2021). The PRISMA 2020 statement: An updated guideline for reporting systematic reviews. BMJ, 372, n71. https://doi.org/10.1136/bmj.n71

Park, S. H., Song, Y. J. C., Demetriou, E. A., Pepper, K. L., Thomas, E. E., Hickie, I. B., \& Guastella, A. J. (2020). Validation of the 21-item depression, anxiety, and stress scales (DASS-21) in individuals with autism spectrum disorder. Psychiatry Research, 291, 113300. https://doi.org/10.1016/j.psychres.2020.113300

Paul, L. K., Corsello, C., Kennedy, D. P., \& Adolphs, R. (2014). Agenesis of the corpus callosum and autism: A comprehensive comparison. Brain: A Journal of Neurology, 137(6), 1813-1829. https://doi.org/10.1093/brain/awu070

Roestorf, A., Bowler, D. M., Deserno, M. K., Howlin, P., Klinger, L., McConachie, H., Parr, J. R., Powell, P., Van Heijst, B. F. C., \& Geurts, H. M. (2019). "Older adults with ASD: The consequences of aging." Insights from a series of special interest group meetings held at the international society for autism research 2016-2017. Research in Autism Spectrum Disorders, 63, 3-12. https://doi.org/ 10.1016/j.rasd.2018.08.007

Rothwell, P. M. (2005). External validity of randomised controlled trials: "To whom do the results of this trial apply?" The Lancet, 365(9453), 82-93. https://doi.org/10.1016/s0140-6736(04) $17670-8$

Russell, G., Mandy, W., Elliott, D., White, R., Pittwood, T., \& Ford, T. (2019). Selection bias on intellectual ability in autism research: A cross-sectional review and meta-analysis. Molecular Autism, 10(1), 1-10. https://doi.org/10.1186/s13229-019-0260-x

Saviola, F., Pappaianni, E., Monti, A., Grecucci, A., Jovicich, J., \& De Pisapia, N. (2020). Trait and state anxiety are mapped differently in the human brain. Scientific Reports, 10(1), 1-11. https://doi. org/10.1038/s41598-020-68008-z

Schulz, K. F., Altman, D. G., \& Moher, D. (2010). CONSORT 2010 statement: Updated guidelines for reporting parallel group randomised trials. Trials, 11(1), 1-8. https://doi.org/10.1186/ 1741-7015-8-18

Sebastian, C., Blakemore, S., \& Charman, T. (2009). Reactions to ostracism in adolescents with autism spectrum conditions. Journal of Autism and Developmental Disorders, 39(8), 1122-1130. https://doi.org/10.1007/s10803-009-0725-4

Simon, D. M., \& Corbett, B. A. (2013). Examining associations between anxiety and cortisol in high functioning male children with autism. Journal of Neurodevelopmental Disorders, 5(1), 1-10. https://doi.org/10.1186/1866-1955-5-32

Sorensen, A. A. M. D., Wojahn, R. D. B. S., Manske, M. C. M. D., \& Calfee, R. P. M. D. M. (2013). Using the strengthening the reporting of observational studies in epidemiology (STROBE) statement to assess reporting of observational trials in hand surgery. The Journal of Hand Surgery, 38(8), 1584-1589. https://doi.org/10. 1016/j.jhsa.2013.05.008

South, M., Carr, A. W., Stephenson, K. G., Maisel, M. E., \& Cox, J. C. (2017). Symptom overlap on the SRS-2 adult self-report between adults with ASD and adults with high anxiety. Autism Research, 10(7), 1215-1220. https://doi.org/10.1002/aur.1764

South, M., Stephenson, K. G., Nielson, C. A., Maisel, M., Top, D. N., \& Kirwan, C. B. (2015). Overactive pattern separation memory associated with negative emotionality in adults diagnosed with autism spectrum disorder. Journal of Autism and Developmental Disorders, 45(11), 3458-3467. https://doi.org/10.1007/ s10803-015-2547-x 
Spain, D., Sin, J., Linder, K. B., McMahon, J., \& Happé, F. (2018). Social anxiety in autism spectrum disorder: A systematic review. Research in Autism Spectrum Disorders, 52, 51-68. https://doi. org/10.1016/j.rasd.2018.04.007

Spence, S. H. (1998). A measure of anxiety symptoms among children. Behaviour Research and Therapy, 36(5), 545-566. https://doi.org/ 10.1016/S0005-7967(98)00034-5

Spielberger, C. (1966). Theory and research on anxiety. In C. Spielberger (Ed.), Anxiety and behavior. Academic press.

Spielberger, C. (1970). Test manual for the state-trait anxiety inventory. Consulting Psychologists Press.

Spielberger, C. (1973). Manual for the state-trait anxiety inventory for children. Consulting Psychologist Press.

Stern, J. A., Gadgil, M. S., Blakeley-Smith, A., Reaven, J. A., \& Hepburn, S. L. (2014). Psychometric properties of the SCARED in youth with autism spectrum disorder. Research in Autism Spectrum Disorders, 8(9), 1225-1234. https://doi.org/10.1016/j.rasd. 2014.06.008

Stratis, E. A., \& Lecavalier, L. (2015). Informant agreement for youth with autism spectrum disorder or intellectual disability: A metaanalysis. Journal of Autism and Developmental Disorders, 45(4), 1026-1041. https://doi.org/10.1007/s10803-014-2258-8

Sweileh, W. M., Al-Jabi, S. W., Sawalha, A. F., \& Zyoud, S. H. (2016). Bibliometric profile of the global scientific research on autism spectrum disorders. Springerplus, 5(1), 1-12. https://doi.org/10. 1186/s40064-016-3165-6

Tan, J., Ma, Z., Gao, X., Wu, Y., \& Fang, F. (2011). Gender difference of unconscious attentional bias in high trait anxiety individuals. PLoS ONE, 6(5), e20305. https://doi.org/10.1371/journal.pone. 0020305

Uljarevic, M., Hedley, D., Rose-Foley, K., Magiati, I., Cai, R. Y., Dissanayake, C., Richdale, A., \& Trollor, J. (2020). Anxiety and depression from adolescence to old age in autism spectrum disorder. Journal of Autism and Developmental Disorders, 50(9), 3155-3165. https://doi.org/10.1007/s10803-019-04084-z

van Steensel, F. J., Bogels, S. M., \& Perrin, S. (2011). Anxiety disorders in children and adolescents with autistic spectrum disorders: A meta-analysis. Clinical Child and Family Psychology Review, 14(3), 302-317. https://doi.org/10.1007/s10567-011-0097-0

van Steensel, F. J. A., \& Heeman, E. J. (2017). Anxiety levels in children with autism spectrum disorder: A meta-analysis. Journal of Child and Family Studies, 26(7), 1753-1767. https://doi.org/10. 1007/s10826-017-0687-7

Vandenbroucke, J. P., Von Elm, E., Altman, D. G., Gøtzsche, P. C., Mulrow, C. D., Pocock, S. J., Poole, C., Schlesselman, J. J., Egger, M., \& Initiative., S. (2007). Strengthening the reporting of observational studies in epidemiology (STROBE): Explanation and elaboration. PLoS Medicine, 4(10), e297. https://doi.org/10. 1371/journal.pmed
Vasa, R. A., Keefer, A., Reaven, J., South, M., \& White, S. W. (2018). Priorities for advancing research on youth with autism spectrum disorder and co-occurring anxiety. Journal of Autism and Developmental Disorders, 48(3), 925-934. https://doi.org/10.1007/ s10803-017-3320-0

Villada, C., Hidalgo, V., Almela, M., \& Salvador, A. (2016). Individual differences in the psychobiological response to psychosocial stress (trier social stress test): The relevance of trait anxiety and coping styles. Stress and Health, 32(2), 90-99. https://doi.org/10.1002/ smi. 2582

von Elm, E., Altman, D. G., Egger, M., Pocock, S. J., Gøtzsche, P. C., \& Vandenbroucke, J. P. (2007). The strengthening the reporting of observational studies in epidemiology (STROBE) statement: Guidelines for reporting observational studies. The Lancet, 370(9596), 1453-1457. https://doi.org/10.1016/s0140-6736(07) 61602-x

White, S. W., Ollendick, T., Scahill, L., Oswald, D., \& Albano, A. M. (2009a). Preliminary efficacy of a cognitive-behavioral treatment program for anxious youth with autism spectrum disorders. Journal of Autism and Developmental Disorders, 39(12), 1652-1662. https://doi.org/10.1007/s10803-009-0801-9

White, S. W., Oswald, D., Ollendick, T., \& Scahill, L. (2009b). Anxiety in children and adolescents with autism spectrum disorders. Clinical Psychology Review, 29(3), 216-229. https://doi.org/10. 1016/j.cpr.2009.01.003

Wong, M. L., Anderson, J., Knorr, T., Joseph, J. W., \& Sanchez, L. D. (2018). Grit, anxiety, and stress in emergency physicians. American Journal of Emergency Medicine, 36(6), 1036-1039. https:// doi.org/10.1016/j.ajem.2018.02.021

Yoo, H.-J., Bahn, G., Cho, I.-H., Kim, E.-K., Kim, J.-H., Min, J.-W., Lee, W.-H., Seo, J.-S., Jun, S.-S., Bong, G., Cho, S., Shin, M.-S., Kim, B.-N., Kim, J.-W., Park, S., \& Laugeson, E. A. (2014). A randomized controlled trial of the Korean version of the PEERS parent-assisted social skills training program for teens with ASD. Autism Research, 7(1), 145-161. https://doi.org/10.1002/aur.1354

Zukerman, G., Yahav, G., \& Ben-Itzchak, E. (2019a). Diametrically opposed associations between academic achievement and social anxiety among university students with and without autism spectrum disorder. Autism Research, 12(9), 1376-1385. https://doi. org/10.1002/aur.2129

Zukerman, G., Yahav, G., \& Ben-Itzchak, E. (2019b). Increased psychiatric symptoms in university students with autism spectrum disorder are associated with reduced adaptive behavior. Psychiatry Research, 273, 732-738. https://doi.org/10.1016/j.psychres.2019. 01.098

Publisher's Note Springer Nature remains neutral with regard to jurisdictional claims in published maps and institutional affiliations. 